\title{
Open string amplitudes of closed topological vertex
}

\author{
Kanehisa Takasaki* \\ Department of Mathematics, Kinki University \\ 3-4-1 Kowakae, Higashi-Osaka, Osaka 577-8502, Japan \\ Toshio Nakatsu ${ }^{\dagger}$ \\ Institute for Fundamental Sciences, Setsunan University \\ 17-8 Ikeda Nakamachi, Neyagawa, Osaka 572-8508, Japan
}

\begin{abstract}
The closed topological vertex is the simplest "off-strip" case of non-compact toric Calabi-Yau threefolds with acyclic web diagrams. By the diagrammatic method of topological vertex, open string amplitudes of topological string theory therein can be obtained by gluing a single topological vertex to an "on-strip" subdiagram of the tree-like web diagram. If non-trivial partitions are assigned to just two parallel external lines of the web diagram, the amplitudes can be calculated with the aid of techniques borrowed from the melting crystal models. These amplitudes are thereby expressed as matrix elements, modified by simple prefactors, of an operator product on the Fock space of $2 \mathrm{D}$ charged free fermions. This fermionic expression can be used to derive $q$-difference equations for generating functions of special subsets of the amplitudes. These $q$-difference equations may be interpreted as the defining equation of a quantum mirror curve.
\end{abstract}

2010 Mathematics Subject Classification: 17B81, 33E20, 81T30

Key words: closed topological vertex, open string amplitude, free fermion, quantum torus, shift symmetry, q-difference equation, mirror curve

\footnotetext{
*E-mail: takasaki@math.h.kyoto-u.ac.jp

${ }^{\dagger}$ E-mail: nakatsu@mpg.setsunan.ac.jp
} 


\section{Introduction}

Topological vertex [1] is a diagrammatic method that captures A-model topological string theory on non-compact toric Calabi-Yau threefolds. In the case of "on-strip" geometry (see Appendix A for a precise setup), this method works particularly well to calculate both the closed string partition function and open string amplitudes in an explicit form [2]. Since the calculation in the on-strip case relies on the linear shape of the toric diagram, it is a technical challenge to extend this result to an "off-strip" case.

The closed topological vertex [3] is one of the simplest examples of "offstrip" geometry. Its web diagram is acyclic (in other words, the threefold has no compact 4-cycle), and the toric diagram has a triangular shape (see Figure 11). The closed string partition function in this case is calculated by several methods including topological vertex [3, 4, 5]. The final expression of the partition function resembles the on-strip case, but the method of derivation is more subtle. Moreover, Karp, Liu and Mariño [4, Section 6.4] argued that such a closed expression of the partition function will cease to exist if branches of the tree-like web diagram are prolonged to arbitrary lengths. In this sense, the closed topological vertex is rather special among off-strip geometry without compact 4-cycle.

In this paper, we calculate open string amplitudes of the closed topological vertex in the case where non-trivial boundary conditions of the string world sheet are imposed on two parallel external lines of the web diagram. Although lacking full generality, this is the first attempt in the literature to calculate open string amplitudes of the closed topological vertex explicitly. Moreover, we use this result to derive $q$-difference equations for generating functions of special subsets of these amplitudes. In the perspectives of mirror geometry of topological string theory [6, 7], the $q$-difference equations may be interpreted as the defining equations of a "quantum mirror curve". This quantum mirror curve will be a new example of quantum curves in the topological recursion program [8].

To calculate the open string amplitudes in question, we use techniques that were developed in our previous work on the melting crystal models [9, 10, 11, 12]. A clue of these techniques is the notion of "shift symmetries" in a quantum torus algebra. This algebra is realized by operators on the Fock space of 2D charged free fermions. The shift symmetries act on a set of basis elements $V_{m}^{(k)}$ of this algebra so as to shift the indices $k, m$ in a certain way. This enables us to relate the commutative subalgebra spanned

\footnotetext{
${ }^{1}$ The same fermionic realization of the quantum torus algebra appears in the work of Okounkov and Pandharipande [13] on the Gromov-Witten invariants of $\mathbb{C P}^{1}$.
} 

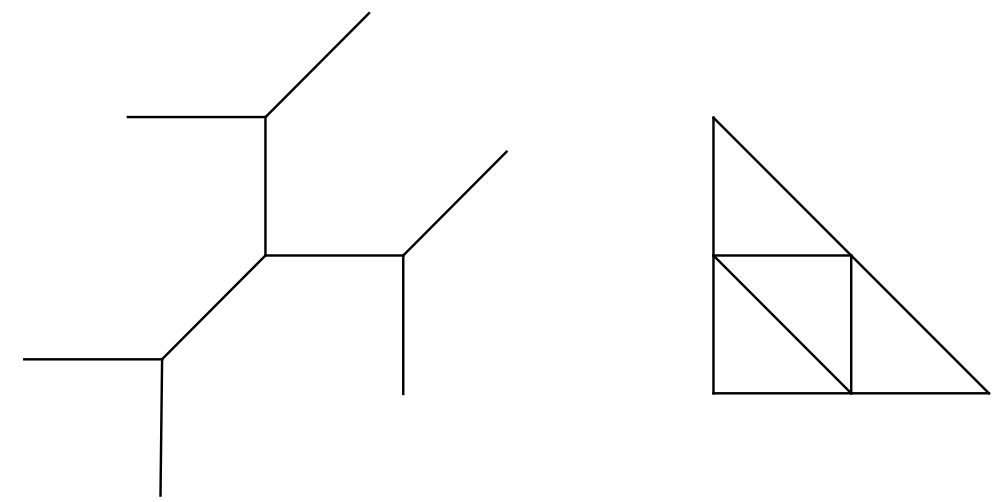

Figure 1: Web diagram (left) and toric diagram (right) of closed topological vertex

by $V_{0}^{(k)}$, 2 to the $U(1)$ current algebra spanned by $V_{m}^{(0)}$ 's. In our previous work, this algebraic machinery is used to convert the partition functions of the melting crystal models to tau functions of the 2D Toda hierarchy. In this paper, we employ the same method to express the open string amplitudes as matrix elements, modified by simple prefactors, of an operator product on the fermionic Fock space.

Our calculation starts from a cut-and-glue description of the amplitude [5]. Namely, the web diagram is cut into two subdiagrams by removing an internal line, and glued together along this line after calculating the contributions of these two parts. One of them is a single topological vertex, and the other is an on-strip diagram for which the result of Iqbal and Kashani-Poor [2] can be used. To glue these two parts again, we have to calculate an infinite sum with respect to a partition on the internal line. This is the place where the aforementioned techniques are used. The amplitude of the closed topological vertex thereby boils down to a product of simple factors and a matrix element of an operator on the Fock space. Moreover, the matrix element turns out to be the open string amplitude of a new on-strip web diagram.

The final expression of the open string amplitudes enables us to derive $q$-difference equations for the generating functions of special subsets of the amplitudes. The generating functions are the Baker-Akhiezer functions in the context of integrable hierarchies, and play the role of "wave functions" of a probe D-brane [6, 7]. Our result is an extension of known results on the resolved conifold [15, 16, 17] and more general on-strip geometry [18]. The structure of the $q$-difference equation is, so to speak, a mixture of the $q$ -

\footnotetext{
${ }^{2}$ Its role as symmetries in the KP and 2D Toda hierarchies was independently studied by Harnad and Orlov [14.
} 
difference equations of the quantum dilogarithmic functions [19, 20] and the $q$-hypergeometric equations that appear in the resolved conifold and more general on-strip geometry. Our result shows that quantum mirror curves beyond on-strip geometry can have an intricate origin.

This paper is organized as follows. In Section 2, the diagrammatic construction of the open string amplitudes are reformulated in a partially summed form. Fermionic tools for the subsequent calculation are also reviewed here. In Section 3, the techniques borrowed from the melting crystal models are used to calculate the amplitudes in terms of fermions. In Section 4, the fermionic expression of the amplitudes is further converted to a final form. A technical clue therein is the cyclic symmetry among "two-leg" topological vertices. This well known symmetry is translated to a kind of "operatorstate correspondence" in the fermionic Fock space, and used to rewrite the fermionic expression of the amplitudes. In Section 5, the generating functions of special subsets of the amplitudes are introduced, and shown to satisfy $q$ difference equations. The structure of the $q$-difference equations is examined in the perspectives of mirror geometry. In Section 6, these results are shown to be consistent with a flop transition. Appendix A is a brief review of the notion of on-strip amplitudes. Appendix B presents another proof of the identities used in Section 4.

\section{Construction of open string amplitudes}

The setup for the open string amplitudes in question is shown in Figure 2. $Q_{1}, Q_{2}, Q_{3}$ are Kähler parameters on the internal lines. $\beta_{1}$ and $\beta_{2}$ are partitions assigned to the two lower external lines. The other external lines are given the trivial partition $\emptyset$. Let $Z_{\beta_{1} \beta_{2}}^{\text {ctv }}$ denote the amplitude in this setup. $Z_{\beta_{1} \beta_{2}}^{\text {ctv }}$ is a sum of weights over all possible values of the partitions $\alpha_{1}, \alpha_{2}, \alpha_{3}$ on the internal lines. The weight for a given configuration of $\alpha_{1}, \alpha_{2}, \alpha_{3}$ is a product of vertex weights and edge weights. These weights depend on the parameter $q$ in the range $|q|<1$.

\subsection{Vertex weights and gluing rules}

The vertex weight at each vertex is the topological vertex 3

$$
C_{\lambda \mu \nu}=q^{\kappa(\mu) / 2} s_{\mathrm{t}_{\nu}}\left(q^{-\rho}\right) \sum_{\eta \in \mathcal{P}} s_{\lambda / \eta}\left(q^{-\nu-\rho}\right) s_{\mu / \eta}\left(q^{-{ }^{\mathrm{t}} \nu-\rho}\right),
$$

\footnotetext{
${ }^{3}$ We follow a definition commonly used in the recent literature 21,22 . This definition differs from the earlier one [1, 6] in that $q$ is replaced by $q^{-1}$ and an overall factor of the form $q^{\kappa(\lambda) / 2+\kappa(\mu) / 2+\kappa(\nu) / 2}$ is multiplied.
} 


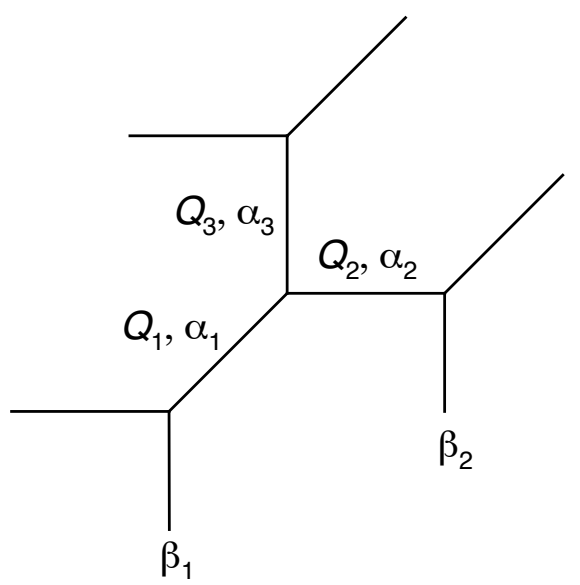

Figure 2: Setup for open string amplitude $Z_{\beta_{1} \beta_{2}}^{\text {ctv }}$

where the sum with respect to $\eta$ ranges over the set $\mathcal{P}$ of all partitions. $\lambda=\left(\lambda_{i}\right)_{i=1}^{\infty}, \mu=\left(\mu_{i}\right)_{i=1}^{\infty}$ and $\nu=\left(\nu_{i}\right)_{i=1}^{\infty}$ are the partitions assigned to the three legs of the vertex that are ordered anti-clockwise, and ${ }^{\mathrm{t}} \nu$ denotes the conjugate (or transposed) partition of $\nu . \kappa(\mu)$ is the second Casimir invariant

$$
\kappa(\mu)=\sum_{i=1}^{\infty} \mu_{i}\left(\mu_{i}-2 i+1\right)=\sum_{i=1}^{\infty}\left(\left(\mu_{i}-i+1 / 2\right)^{2}-(-i+1 / 2)^{2}\right) .
$$

$s_{\mathrm{t}_{\nu}}\left(q^{-\rho}\right), s^{\mathrm{t}_{\lambda / \eta}}\left(q^{-\nu-\rho}\right)$ and $s_{\mu / \eta}\left(q^{{ }^{\mathrm{t}} \nu-\rho}\right)$ are special values of the infinitevariate Schur function $s_{t_{\nu}}(\boldsymbol{x})$ and the skew Schur functions $s_{{ }}{ }_{\lambda / \eta}(\boldsymbol{x}), s_{\mu / \eta}(\boldsymbol{x})$, $\boldsymbol{x}=\left(x_{1}, x_{2}, \ldots\right)$, at

$$
q^{-\rho}=\left(q^{i-1 / 2}\right)_{i=1}^{\infty}, \quad q^{-\nu-\rho}=\left(q^{-\nu_{i}+i-1 / 2}\right)_{i=1}^{\infty}, \quad q^{{ }^{-\mathrm{t}} \nu-\rho}=\left(q^{-{ }^{\mathrm{t}} \nu_{i}+i-1 / 2}\right)_{i=1}^{\infty} .
$$

The vertex weight enjoy the cyclic symmetry

$$
C_{\lambda \mu \nu}=C_{\mu \nu \lambda}=C_{\nu \lambda \mu}
$$

that can be deduced from the crystal interpretation of the vertex weight [23].

The vertex weights $C_{\lambda \mu \nu}$ and $C_{\lambda^{\prime} \mu^{\prime} \nu^{\prime}}$ at two vertices connecting an internal line are glued together by the following rules:

(i) The partitions on the internal line, say $\lambda$ and $\lambda^{\prime}$, are matched as

$$
\lambda^{\prime}={ }^{\mathrm{t}} \lambda \text {. }
$$

(ii) The product of the vertex weights is multiplied by the edge weight

$$
(-Q)^{|\lambda|}(-1)^{n|\lambda|} q^{-n \kappa(\lambda) / 2},
$$




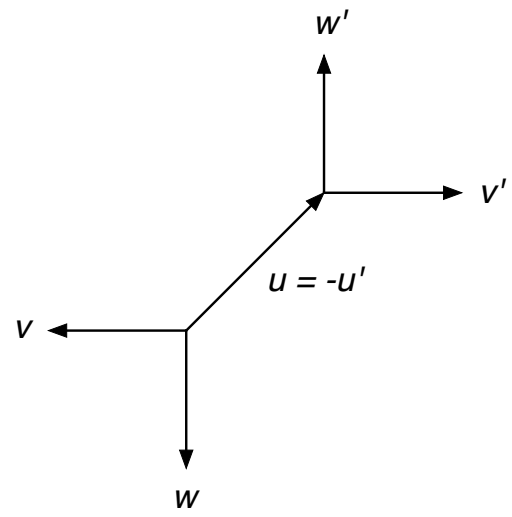

Figure 3: Configuration of vectors that determine the framing number

where $Q$ is the Kähler parameter of the internal line, and $n$ is an integer called "the framing number".

The framing number is defined as

$$
n=v^{\prime} \wedge v=w^{\prime} \wedge w,
$$

where $v, w$ and $v^{\prime}, w^{\prime}$ are vectors in the web diagram that emanate from the two vertices (see Figure 31). The wedge product means the determinant of the $2 \times 2$ matrix formed by the two vectors, i.e.,

$$
v^{\prime} \wedge v=v_{1}^{\prime} v_{2}-v_{2}^{\prime} v_{1}
$$

for $v^{\prime}=\left(v_{1}^{\prime}, v_{2}^{\prime}\right)$ and $v=\left(v_{1}, v_{2}\right)$. These vectors $v, w$ and $v^{\prime}, w^{\prime}$ are chosen along with the third vectors $u, u^{\prime}, u+u^{\prime}=0$, in such a way that $u, v, w$ and $u^{\prime}, v^{\prime}, w^{\prime}$ are ordered anti-clockwise and satisfy the zero-sum relations

$$
u+v+w=0, \quad u^{\prime}+v^{\prime}+w^{\prime}=0 .
$$

These sets of vectors are uniquely determined as far as the toric diagram is fully triangulated (i.e., the area of each triangle is $1 / 2$ ).

\subsection{Reformulation of amplitude}

The amplitude $Z_{\beta_{1} \beta_{2}}^{\text {ctv }}$ is given by a sum of the product of these weights over $\alpha_{1}, \alpha_{2}, \alpha_{3} \in \mathcal{P}$. Following Sułkowski's formulation [5], we decompose this sum to a partial with sum respect to $\alpha_{1}, \alpha_{2}$ at the first stage and a sum with respect to $\alpha_{3}$ at the next stage. The full amplitude can be thus reformulated as

$$
Z_{\beta_{1} \beta_{2}}^{\mathrm{ctv}}=\sum_{\alpha_{3} \in \mathcal{P}} Z_{\beta_{1} \beta_{2} \mid \alpha_{3}}\left(-Q_{3}\right)^{\left|\alpha_{3}\right|} C_{\mathrm{t}_{\alpha_{3}} \emptyset \emptyset}
$$



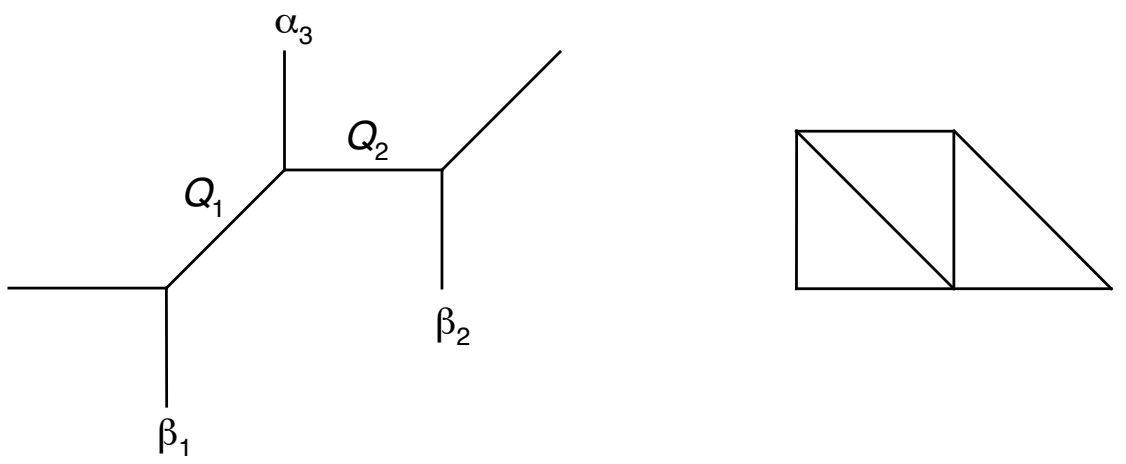

Figure 4: Double- $\mathbb{P}^{1}$ diagram defining $Z_{\beta_{1} \beta_{2} \mid \alpha_{3}}$

$Z_{\beta_{1} \beta_{2} \mid \alpha_{3}}$ is the partial sum with respect to $\alpha_{1}, \alpha_{2}$ and represents the contribution from the lower part of the web diagram. This part is glued with the upper part via the internal line carrying $\alpha_{3}$. $\left(-Q_{3}\right)^{\left|\alpha_{3}\right|}$ is the edge weight of this internal line. Note that the framing number (2.3) in this case is equal to $0 . C_{\mathrm{t}} \emptyset \emptyset$ is the contribution from the upper part of the web diagram. By the cyclic symmetry (2.2), this vertex weight reduces to a special value of the Schur function:

$$
C_{\mathrm{t}_{\alpha_{3} \emptyset \emptyset}}=C_{\emptyset \emptyset \mathrm{t}} \alpha_{3}=s_{\alpha_{3}}\left(q^{-\rho}\right) .
$$

The partial sum $Z_{\beta_{1} \beta_{2} \mid \alpha_{3}}$ itself may be thought of as an open string amplitude of the web diagram (called "double- $\mathbb{P}^{1 "}$ ) shown in Figure 4, Since this is a diagram "on a strip", the associated open string amplitude can be calculated by the well known result [2] (see Appendix A):

$$
\begin{aligned}
Z_{\beta_{1} \beta_{2} \mid \alpha_{3}}= & s_{{ }_{\beta_{1}}}\left(q^{-\rho}\right) s_{{ }_{\beta_{2}}}\left(q^{-\rho}\right) s_{{ }^{\mathrm{t}} \alpha_{3}}\left(q^{-\rho}\right) \prod_{i, j=1}^{\infty}\left(1-Q_{1} Q_{2} q^{-\beta_{1 i}-{ }^{\mathrm{t}} \beta_{2 j}+i+j-1}\right)^{-1} \\
& \times \prod_{i, j=1}^{\infty}\left(1-Q_{1} q^{-\beta_{1 i}-\alpha_{3 j}+i+j-1}\right) \prod_{i, j=1}^{\infty}\left(1-Q_{2} q^{-{ }^{\mathrm{t}} \alpha_{3 i}-{ }^{\mathrm{t}} \beta_{2 j}+i+j-1}\right) .
\end{aligned}
$$

Plugging these building blocks into (2.4), we obtain the following expres- 
sion of $Z_{\beta_{1} \beta_{2}}^{\mathrm{ctv}}$ :

$$
\begin{aligned}
Z_{\beta_{1} \beta_{2}}^{\mathrm{ctv}}= & s{ }_{\mathrm{t}_{1}}\left(q^{-\rho}\right) s_{\mathrm{t}_{\beta_{2}}}\left(q^{-\rho}\right) \prod_{i, j=1}^{\infty}\left(1-Q_{1} Q_{2} q^{-\beta_{1 i}-{ }^{\mathrm{t}} \beta_{2 j}+i+j-1}\right)^{-1} \\
& \times \sum_{\alpha_{3} \in \mathcal{P}} s_{{ }^{\mathrm{t}} \alpha_{3}}\left(q^{-\rho}\right) s_{\alpha_{3}}\left(q^{-\rho}\right)\left(-Q_{3}\right)^{\left|\alpha_{3}\right|} \prod_{i, j=1}^{\infty}\left(1-Q_{1} q^{-\beta_{1 i}-\alpha_{3 j}+i+j-1}\right) \\
& \times \prod_{i, j=1}^{\infty}\left(1-Q_{2} q^{-{ }^{\mathrm{t}} \alpha_{3 i}-{ }^{\mathrm{t}} \beta_{2 j}+i+j-1}\right) .
\end{aligned}
$$

Note here that the sum with respect to $\alpha_{3}$ resembles the partition function of the modified melting model [11, 12]: The main part of the Boltzmann weight therein takes the product form $s_{{ }^{t} \alpha_{3}}\left(q^{-\rho}\right) s_{\alpha_{3}}\left(q^{-\rho}\right)$, and this weight is deformed by external potentials depending on $\alpha_{3}$. To calculate this sum, we use the machinery of $2 \mathrm{D}$ charged free fermions.

\subsection{Fermionic Fock space and operators}

The setup of the fermionic Fock space and operators is the same as used for the melting crystal models [9, 10, 11, 12]. Let $\psi_{n}, \psi_{n}^{*}, n \in \mathbb{Z}$, denote the Fourier modes of the 2D charged free fermion fields $\psi(z), \psi^{*}(z)$. They satisfy the anti-commutation relations

$$
\psi_{m} \psi_{n}^{*}+\psi_{n}^{*} \psi_{m}=\delta_{m+n, 0}, \quad \psi_{m} \psi_{n}+\psi_{n} \psi_{m}=0, \quad \psi_{m}^{*} \psi_{n}^{*}+\psi_{n}^{*} \psi_{m}^{*}=0 .
$$

The associated Fock space and its dual space are decomposed to the charge$s$ sectors for $s \in \mathbb{Z}$. It is only the charge- 0 sector that is relevant to the calculation of (2.7). An orthonormal basis of the charge-0 sector is given the ground states

$$
\begin{aligned}
\langle 0| & =\langle-\infty| \cdots \psi_{-i+1}^{*} \cdots \psi_{-1}^{*} \psi_{0}^{*}, \\
|0\rangle & =\psi_{0} \psi_{1} \cdots \psi_{i-1} \cdots|-\infty\rangle
\end{aligned}
$$

and the excited states

$$
\begin{aligned}
& \langle\lambda|=\langle-\infty| \cdots \psi_{\lambda_{i}-i+1}^{*} \cdots \psi_{\lambda_{2}-1}^{*} \psi_{\lambda_{1}}^{*}, \\
& |\lambda\rangle=\psi_{-\lambda_{1}} \psi_{-\lambda_{2}+1} \cdots \psi_{-\lambda_{i}+i-1} \cdots|-\infty\rangle
\end{aligned}
$$

labelled by partitions. The normal ordered product $: \psi_{m} \psi_{n}^{*}$ : is defined as

$$
: \psi_{m} \psi_{n}^{*}:=\psi_{m} \psi_{n}^{*}-\left\langle 0\left|\psi_{m} \psi_{n}^{*}\right| 0\right\rangle
$$

The following operators on the Fock space are used as fundamental tools in our calculation. 
(i) The zero-modes

$$
L_{0}=\sum_{n \in \mathbb{Z}} n: \psi_{-n} \psi_{n}^{*}:, \quad W_{0}=\sum_{n \in \mathbb{Z}} n^{2}: \psi_{-n} \psi_{n}^{*}:
$$

of the Virasoro and $W_{3}$ algebras and the Fourier modes

$$
J_{m}=\sum_{n \in \mathbb{Z}}: \psi_{-n} \psi_{n+m}^{*}:, \quad m \in \mathbb{Z},
$$

of the fermionic current : $\psi(z) \psi^{*}(z)$ :

(ii) The fermionic realization

$$
K=\sum_{n \in \mathbb{Z}}(n-1 / 2)^{2}: \psi_{-n} \psi_{n}^{*}:=W_{0}-L_{0}+J_{0} / 4
$$

of the so called "cut-and-join operator" [24, 25].

(iii) The basis elements

$$
V_{m}^{(k)}=q^{-k m / 2} \sum_{n \in \mathbb{Z}} q^{k n}: \psi_{m-n} \psi_{n}^{*}:, \quad k, m \in \mathbb{Z},
$$

of a fermionic realization of the quantum torus algebra [9, 13].

(iv) The vertex operators [26, 27]

$$
\Gamma_{ \pm}(z)=\exp \left(\sum_{k=1}^{\infty} \frac{z^{k}}{k} J_{ \pm k}\right), \quad \Gamma_{ \pm}^{\prime}(z)=\exp \left(-\sum_{k=1}^{\infty} \frac{(-z)^{k}}{k} J_{ \pm k}\right)
$$

and the multi-variable extensions

$$
\Gamma_{ \pm}(\boldsymbol{x})=\prod_{i \geq 1} \Gamma_{ \pm}\left(x_{i}\right), \quad \Gamma_{ \pm}^{\prime}(\boldsymbol{x})=\prod_{i \geq 1} \Gamma_{ \pm}^{\prime}\left(x_{i}\right) .
$$

The matrix elements of these operators are well known. $J_{0}, L_{0}, W_{0}, K$ are diagonal with respect to the basis $\{|\lambda\rangle\}_{\lambda \in \mathcal{P}}$ in the charge- 0 sector:

$$
\begin{gathered}
\left\langle\lambda\left|J_{0}\right| \mu\right\rangle=0, \quad\left\langle\lambda\left|L_{0}\right| \mu\right\rangle=\delta_{\lambda \mu}|\lambda|, \\
\left\langle\lambda\left|W_{0}\right| \mu\right\rangle=\delta_{\lambda \mu}(\kappa(\lambda)+|\lambda|), \quad\langle\lambda|K| \mu\rangle=\delta_{\lambda \mu} \kappa(\lambda) .
\end{gathered}
$$

The matrix elements of $\Gamma_{ \pm}(\boldsymbol{x})$ and $\Gamma_{ \pm}^{\prime}(\boldsymbol{x})$ are skew Schur functions [28, 29]:

$$
\begin{gathered}
\left\langle\lambda\left|\Gamma_{-}(\boldsymbol{x})\right| \mu\right\rangle=\left\langle\mu\left|\Gamma_{+}(\boldsymbol{x})\right| \lambda\right\rangle=s_{\lambda / \mu}(\boldsymbol{x}), \\
\left\langle\lambda\left|\Gamma_{-}^{\prime}(\boldsymbol{x})\right| \mu\right\rangle=\left\langle\mu\left|\Gamma_{+}^{\prime}(\boldsymbol{x})\right| \lambda\right\rangle=s^{{ }^{\mathrm{t}}{ }_{\lambda /{ }^{\mathrm{t}} \mu}(\boldsymbol{x}) .}
\end{gathered}
$$




\section{Calculation of sum in (2.7)}

Let us proceed to calculation of the sum in (2.7). This comprises two steps. In the first step, we express $s_{\mathrm{t}_{\alpha_{3}}}\left(q^{-\alpha}\right)$ and $s_{\alpha_{3}}\left(q^{-\rho}\right)$ in a fermionic form, and convert the c-number factors $\prod_{i, j=1}^{\infty}\left(1-Q_{1} \cdots\right)$ and $\prod_{i, j=1}^{\infty}\left(1-Q_{2} \cdots\right)$ to operators inserted in the fermionic expression of the Schur functions. The sum with respect to $\alpha_{3}$ thereby turns into the vacuum expectation value of an operator product on the Fock space. In the second step, we use the "shift symmetries" of the quantum torus algebra [9, 10, 11, 12] to rewrite the vacuum expectation value further. This calculation is more or less parallel to the way the partition functions of the various melting crystal models are converted to tau functions of the 2D Toda hierarchy.

\subsection{Step 1: Translation to fermionic language}

The infinite products $\prod_{i, j}^{\infty}\left(1-Q_{1} \cdots\right)$ and $\prod_{i, j=1}^{\infty}\left(1-Q_{2} \cdots\right)$ can be reexpressed in an exponential form as

$$
\prod_{i, j=1}^{\infty}\left(1-Q_{1} q^{-\beta_{1 i}-\alpha_{3 j}+i+j-1}\right)=\exp \left(-\sum_{i, k=1}^{\infty} \frac{\left(Q_{1} q^{-\beta_{1 i}+i}\right)^{k}}{k} \sum_{j=1}^{\infty} q^{-k\left(\alpha_{3 j}-j+1\right)}\right)
$$

and

$$
\prod_{i, j=1}^{\infty}\left(1-Q_{2} q^{-{ }^{\mathrm{t}} \alpha_{3 i}-{ }^{\mathrm{t}} \beta_{2 j}+i+j-1}\right)=\exp \left(-\sum_{j, k=1}^{\infty} \frac{\left(Q_{2} q^{-{ }^{\mathrm{t}} \beta_{2 j}+j}\right)^{k}}{k} \sum_{i=1}^{\infty} q^{-k\left({ }^{\mathrm{t}} \alpha_{3 i}-i+1\right)}\right) .
$$

We convert these c-number factors to operators inserted in the fermionic expression

$$
s_{{ } \alpha_{3}}\left(q^{-\rho}\right)=\left\langle 0\left|\Gamma_{+}^{\prime}\left(q^{-\rho}\right)\right| \alpha_{3}\right\rangle, \quad s_{\alpha_{3}}\left(q^{-\rho}\right)=\left\langle\alpha_{3}\left|\Gamma_{-}\left(q^{-\rho}\right)\right| 0\right\rangle
$$

of the special values of the Schur functions.

To this end, let us note that $\sum_{j=1}^{\infty} q^{-k\left(\alpha_{3 j}-j+1\right)}$ and $\sum_{j=1}^{\infty} q^{-k\left({ }^{\mathrm{t}} \alpha_{3 i}-i+1\right)}$ are related to eigenvalues of $V_{0}^{( \pm k)}$, s as shown below.

Lemma 1. For any $k>0$ and any $\lambda \in \mathcal{P}$,

$$
\begin{aligned}
\left(V_{0}^{(-k)}+\frac{1}{1-q^{k}}\right)|\lambda\rangle & =\sum_{i=1}^{\infty} q^{-k\left(\lambda_{i}-i+1\right)}|\lambda\rangle \\
\left(V_{0}^{(k)}-\frac{q^{k}}{1-q^{k}}\right)|\lambda\rangle & =-q^{k} \sum_{i=1}^{\infty} q^{-k\left({ }^{t} \lambda_{i}-i+1\right)}|\lambda\rangle .
\end{aligned}
$$


Remark 1. (3.1) and (3.2) imply the relations

$$
\begin{aligned}
\langle\lambda|\left(V_{0}^{(-k)}+\frac{1}{1-q^{k}}\right) & =\langle\lambda| \sum_{i=1}^{\infty} q^{-k\left(\lambda_{i}-i+1\right)}, \\
\langle\lambda|\left(V_{0}^{(k)}-\frac{q^{k}}{1-q^{k}}\right) & =-\langle\lambda| q^{k} \sum_{i=1}^{\infty} q^{-k\left({ }^{\mathrm{t}} \lambda_{i}-i+1\right)}
\end{aligned}
$$

in the dual Fock space as well.

Proof. It is straightforward to derive (3.1):

$$
\begin{aligned}
V_{0}^{(-k)}|\lambda\rangle & =\sum_{j=1}^{\infty}\left(q^{-k\left(\lambda_{j}-j+1\right)}-q^{-k(-j+1)}\right)|\lambda\rangle \\
& =\left(\sum_{j=1}^{\infty} q^{-k\left(\lambda_{j}-j+1\right)}-\frac{1}{1-q^{k}}\right)|\lambda\rangle
\end{aligned}
$$

The subtraction term $q^{-k(-j+1)}$ in this calculation originates in the normal ordering

$$
: \psi_{-n} \psi_{n}^{*}:= \begin{cases}\psi_{-n} \psi_{n}^{*} & \text { for } n>0 \\ \psi_{-n} \psi_{n}^{*}-1 & \text { for } n \leq 0\end{cases}
$$

It is not straightforward to derive (3.2). Let $n$ be an integer greater than or equal to the length of $\lambda$. Accordingly, $\lambda_{i}=i$ for $i>n$. Since the set of all integers $i \leq n$ can be divided into two disjoint sets as

$$
\{i \mid i \leq n\}=\left\{{ }^{\mathrm{t}} \lambda_{i}-i+1 \mid i \geq 1\right\} \cup\left\{-\lambda_{i}+i \mid 1 \leq i \leq n\right\},
$$

one obtains the identity

$$
\sum_{i=1}^{\infty} q^{-k\left({ }^{\mathrm{t}} \lambda_{i}-i+1\right)}+\sum_{i=1}^{n} q^{-k\left(-\lambda_{i}+i\right)}=\sum_{i=-\infty}^{n} q^{-k i}=\frac{q^{-k n}}{1-q^{k}},
$$

which implies that

$$
\begin{aligned}
\sum_{i=1}^{\infty} q^{-k\left({ }^{\mathrm{t}} \lambda_{i}-i+1\right)} & =-\sum_{i=1}^{n} q^{-k\left(-\lambda_{i}+i\right)}+\frac{q^{-k n}}{1-q^{k}} \\
& =-\sum_{i=1}^{n}\left(q^{-k\left(-\lambda_{i}+i\right)}-q^{-k i}\right)+\frac{1}{1-q^{k}} \\
& =-q^{-k} \sum_{i=1}^{n}\left(q^{k\left(\lambda_{i}-i+1\right)}-q^{k(-i+1)}\right)+\frac{1}{1-q^{k}}
\end{aligned}
$$


Consequently,

$$
\begin{aligned}
V_{0}^{(k)}|\lambda\rangle & =\sum_{i=1}^{n}\left(q^{k\left(\lambda_{i}-i+1\right)}-q^{k(-i+1)}\right)|\lambda\rangle \\
& =\left(-q^{k} \sum_{i=1}^{\infty} q^{-k\left({ }^{\mathrm{t}} \lambda_{i}-i+1\right)}+\frac{q^{k}}{1-q^{k}}\right)|\lambda\rangle .
\end{aligned}
$$

(3.2) can be thus derived.

By (3.1) and (3.2), the c-number factors $\prod_{i, j=1}^{\infty}\left(1-Q_{1} \cdots\right)$ and $\prod_{i, j=1}^{\infty}(1-$ $\left.Q_{2} \cdots\right)$ can be converted to operators on the Fock space as

$$
\begin{aligned}
& \prod_{i, j=1}^{\infty}\left(1-Q_{1} q^{-\beta_{1 i}-\alpha_{3 j}+i+j-1}\right) \cdot s_{{ }{ }_{\alpha 3}}\left(q^{-\rho}\right) \\
& =\left\langle 0\left|\Gamma_{+}^{\prime}\left(q^{-\rho}\right) \exp \left(-\sum_{i, k=1}^{\infty} \frac{\left(Q_{1} q^{-\beta_{1 i}+i}\right)^{k}}{k}\left(V_{0}^{(-k)}+\frac{1}{1-q^{k}}\right)\right)\right| \alpha_{3}\right\rangle
\end{aligned}
$$

and

$$
\begin{aligned}
& \prod_{i, j=1}^{\infty}\left(1-Q_{2} q^{-{ }^{\mathrm{t}} \alpha_{3 i}-{ }^{\mathrm{t}} \beta_{2 j}+i+j-1}\right) \cdot s_{\alpha_{3}}\left(q^{-\rho}\right) \\
& =\left\langle\alpha_{3}\left|\exp \left(\sum_{j, k=1}^{\infty} \frac{\left(Q_{2} q^{-{ }^{\mathrm{t}} \beta_{2 j}+j-1}\right)^{k}}{k}\left(V_{0}^{(k)}-\frac{q^{k}}{1-q^{k}}\right)\right) \Gamma_{-}\left(q^{-\rho}\right)\right| 0\right\rangle .
\end{aligned}
$$

Moreover, the factor $\left(-Q_{3}\right)^{\left|\alpha_{3}\right|}$ can be identified with the diagonal matrix element of $\left(-Q_{3}\right)^{L_{0}}$. Having derived these building blocks, we can now use the partition of unity

$$
\sum_{\alpha_{3} \in \mathcal{P}}\left|\alpha_{3}\right\rangle\left\langle\alpha_{3}\right|=1
$$

in the charge- 0 sector to rewrite the sum in (2.7) to the vacuum expectation value of an operator product:

$$
\begin{aligned}
& \sum_{\alpha_{3} \in \mathcal{P}} s_{{ }^{{ }} \alpha_{3}}\left(q^{-\rho}\right) s_{\alpha_{3}}\left(q^{-\rho}\right)\left(-Q_{3}\right)^{\left|\alpha_{3}\right|} \prod_{i, j=1}^{\infty}\left(1-Q_{1} \cdots\right) \prod_{i, j=1}^{\infty}\left(1-Q_{2} \cdots\right) \\
& =\langle 0| \Gamma_{+}^{\prime}\left(q^{-\rho}\right) \exp \left(-\sum_{i, k=1}^{\infty} \frac{\left(Q_{1} q^{-\beta_{1 i}+i}\right)^{k}}{k}\left(V_{0}^{(-k)}+\frac{1}{1-q^{k}}\right)\right)\left(-Q_{3}\right)^{L_{0}} \\
& \quad \times \exp \left(\sum_{j, k=1}^{\infty} \frac{\left(Q_{2} q^{-{ }^{t} \beta_{2 j}+j-1}\right)^{k}}{k}\left(V_{0}^{(k)}-\frac{q^{k}}{1-q^{k}}\right)\right) \Gamma_{-}\left(q^{-\rho}\right)|0\rangle .
\end{aligned}
$$




\subsection{Step 2: Use of shift symmetries}

Let us recall the following consequence of the shift symmetries of the quantum torus algebra [9, 10, 11. Note that the last one (3.8) is modified from the previous formulation in terms of $W_{0}$.

\section{Lemma 2.}

$$
\begin{gathered}
\Gamma_{-}^{\prime}\left(q^{-\rho}\right) \Gamma_{+}^{\prime}\left(q^{-\rho}\right)\left(V_{0}^{(-k)}+\frac{1}{1-q^{k}}\right)=V_{k}^{(-k)} \Gamma_{-}^{\prime}\left(q^{-\rho}\right) \Gamma_{+}^{\prime}\left(q^{-\rho}\right), \\
\left(V_{0}^{(k)}-\frac{q^{k}}{1-q^{k}}\right) \Gamma_{-}\left(q^{-\rho}\right) \Gamma_{+}\left(q^{-\rho}\right)=\Gamma_{-}\left(q^{-\rho}\right) \Gamma_{+}\left(q^{-\rho}\right)(-1)^{k} V_{-k}^{(k)}, \\
V_{k}^{(-k)}=q^{-k / 2} q^{K / 2} J_{k} q^{-K / 2}, \quad V_{-k}^{(k)}=q^{k / 2} q^{K / 2} J_{-k} q^{-K / 2} .
\end{gathered}
$$

We use these operator identities to rewrite (3.5) further. Let us first examine the left side of $\left(-Q_{3}\right)^{L_{3}}$ in (3.5) . Upon inserting $q^{-K / 2} \Gamma_{-}^{\prime}\left(q^{-\rho}\right)$ to the right of $\langle 0|$ as

$$
\langle 0| \Gamma_{+}^{\prime}\left(q^{-\rho}\right)=\langle 0| q^{-K / 2} \Gamma_{-}^{\prime}\left(q^{-\rho}\right) \Gamma_{+}^{\prime}\left(q^{-\rho}\right),
$$

we can use (3.6) and (3.8) to rewrite the left side of $\left(-Q_{3}\right)^{L_{3}}$ as

$$
\begin{aligned}
& \langle 0| \Gamma_{+}^{\prime}\left(q^{-\rho}\right) \exp \left(-\sum_{i, k=1}^{\infty} \frac{\left(Q_{1} q^{-\beta_{1 i}+i}\right)^{k}}{k}\left(V_{0}^{(-k)}+\frac{1}{1-q^{k}}\right)\right) \\
& =\langle 0| q^{-K / 2} \exp \left(-\sum_{i, k=1}^{\infty} \frac{\left(Q_{1} q^{-\beta_{1 i}+i}\right)^{k}}{k} V_{k}^{(-k)}\right) \Gamma_{-}^{\prime}\left(q^{-\rho}\right) \Gamma_{+}^{\prime}\left(q^{-\rho}\right) \\
& =\langle 0| \exp \left(-\sum_{i, k=1}^{\infty} \frac{\left(Q_{1} q^{-\beta_{1 i}+i}\right)^{k}}{k} q^{-k / 2} J_{k}\right) q^{-K / 2} \Gamma_{-}^{\prime}\left(q^{-\rho}\right) \Gamma_{+}^{\prime}\left(q^{-\rho}\right) \\
& =\langle 0| \exp \left(-\sum_{i, k=1}^{\infty} \frac{\left(Q_{1} q^{-\beta_{1 i}+i-1 / 2}\right)^{k}}{k} J_{k}\right) q^{-K / 2} \Gamma_{-}^{\prime}\left(q^{-\rho}\right) \Gamma_{+}^{\prime}\left(q^{-\rho}\right) .
\end{aligned}
$$

The exponential operator in the last line is essentially a vertex operator,

$$
\exp \left(-\sum_{i, k=1}^{\infty} \frac{\left(Q_{1} q^{-\beta_{1 i}+i-1 / 2}\right)^{k}}{k} J_{k}\right)=\left(-Q_{1}\right)^{-L_{0}} \Gamma_{+}^{\prime}\left(q^{-\beta_{1}-\rho}\right)\left(-Q_{1}\right)^{L_{0}}
$$

hence

$$
\begin{aligned}
& \langle 0| \Gamma_{+}^{\prime}\left(q^{-\rho}\right) \exp \left(-\sum_{i, k=1}^{\infty} \frac{\left(Q_{1} q^{-\beta_{1 i}+i}\right)^{k}}{k}\left(V_{0}^{(-k)}+\frac{1}{1-q^{k}}\right)\right) \\
& =\langle 0| \Gamma_{+}^{\prime}\left(q^{-\beta_{1}-\rho}\right)\left(-Q_{1}\right)^{L_{0}} q^{-K / 2} \Gamma_{-}^{\prime}\left(q^{-\rho}\right) \Gamma_{+}^{\prime}\left(q^{-\rho}\right) .
\end{aligned}
$$


In exactly the same manner, using (3.7) and (3.8), we can rewrite the right side of $\left(-Q_{3}\right)^{L_{0}}$ as

$$
\begin{aligned}
& \exp \left(\sum_{j, k=1}^{\infty} \frac{\left(Q_{2} q^{-{ }^{\mathrm{t}} \beta_{2 j}+j-1}\right)^{k}}{k}\left(V_{0}^{(k)}-\frac{q^{k}}{1-q^{k}}\right)\right) \Gamma_{-}\left(q^{-\rho}\right)|0\rangle \\
& =\Gamma_{-}\left(q^{-\rho}\right) \Gamma_{+}\left(q^{-\rho}\right) q^{K / 2}\left(-Q_{2}\right)^{L_{0}} \Gamma_{-}\left(q^{-{ }^{\mathrm{t}} \beta_{2}-\rho}\right)|0\rangle .
\end{aligned}
$$

Plugging (3.9) and (3.10) into (3.5) yields the following expression of the sum in (2.7):

$$
\begin{aligned}
& \sum_{\alpha_{3} \in \mathcal{P}} s_{{ }^{t} \alpha_{3}}\left(q^{-\rho}\right) s_{\alpha_{3}}\left(q^{-\rho}\right)\left(-Q_{3}\right)^{\left|\alpha_{3}\right|} \prod_{i, j=1}^{\infty}\left(1-Q_{1} \cdots\right) \prod_{i, j=1}^{\infty}\left(1-Q_{2} \cdots\right) \\
& =\langle 0| \Gamma_{+}^{\prime}\left(q^{-\beta_{1}-\rho}\right)\left(-Q_{1}\right)^{L_{0}} q^{-K / 2} \Gamma_{-}^{\prime}\left(q^{-\rho}\right) \Gamma_{+}^{\prime}\left(q^{-\rho}\right)\left(-Q_{3}\right)^{L_{0}} \\
& \quad \times \Gamma_{-}\left(q^{-\rho}\right) \Gamma_{+}\left(q^{-\rho}\right) q^{K / 2}\left(-Q_{2}\right)^{L_{0}} \Gamma_{-}\left(q^{-{ }^{\mathrm{t}} \beta_{2}-\rho}\right)|0\rangle .
\end{aligned}
$$

\section{Final expression of open string amplitudes}

We have thus derived the following intermediate expression of $Z_{\beta_{1} \beta_{2}}^{\mathrm{ctv}}$ :

$$
\begin{aligned}
Z_{\beta_{1} \beta_{2}}^{\mathrm{ctv}}= & s_{\mathrm{t}_{\beta_{1}}}\left(q^{-\rho}\right) s_{\mathrm{t}_{\beta_{2}}}\left(q^{-\rho}\right) \prod_{i, j=1}^{\infty}\left(1-Q_{1} Q_{2} q^{-\beta_{1 i}{ }^{-\mathrm{t}} \beta_{2 j}+i+j-1}\right)^{-1} \\
& \times\langle 0| \Gamma_{+}^{\prime}\left(q^{-\beta_{1}-\rho}\right)\left(-Q_{1}\right)^{L_{0}} q^{-K / 2} \Gamma_{-}^{\prime}\left(q^{-\rho}\right) \Gamma_{+}^{\prime}\left(q^{-\rho}\right)\left(-Q_{3}\right)^{L_{0}} \\
& \times \Gamma_{-}\left(q^{-\rho}\right) \Gamma_{+}\left(q^{-\rho}\right) q^{K / 2}\left(-Q_{2}\right)^{L_{0}} \Gamma_{-}\left(q^{-{ }^{\mathrm{t}} \beta_{2}-\rho}\right)|0\rangle .
\end{aligned}
$$

As a final step, we use the following relations in the fermionic Fock space that can be derived from a special case of the cyclic symmetry (2.2). This is a kind of operator-state correspondence that maps vertex operators of the form $\Gamma_{ \pm}\left(q^{-{ }^{t} \lambda-\rho}\right)$ and $\Gamma_{ \pm}^{\prime}\left(q^{-\lambda-\rho}\right)$ to the state vectors $\left\langle{ }^{\mathrm{t}} \lambda\right|$ and $\left|{ }^{\mathrm{t}} \lambda\right\rangle$ in the Fock space.

Lemma 3. For any $\lambda \in \mathcal{P}$,

$$
\begin{aligned}
s_{{ }}\left(q^{-\rho}\right) \Gamma_{-}^{\prime}\left(q^{-\lambda-\rho}\right)|0\rangle & =q^{K / 2} \Gamma_{-}\left(q^{-\rho}\right) \Gamma_{+}\left(q^{-\rho}\right)\left|{ }^{\mathrm{t}} \lambda\right\rangle, \\
s_{{ }{ }_{\lambda}}\left(q^{-\rho}\right) \Gamma_{-}\left(q^{-{ }^{\mathrm{t}} \lambda-\rho}\right)|0\rangle & =q^{\kappa(\lambda) / 2} q^{-K / 2} \Gamma_{-}^{\prime}\left(q^{-\rho}\right) \Gamma_{+}^{\prime}\left(q^{-\rho}\right)\left|{ }^{\mathrm{t}} \lambda\right\rangle .
\end{aligned}
$$

Remark 2. There are a number of apparently different, but equivalent forms of these relations. For example, one can use the well known identity [28]

$$
s_{{ }_{\lambda}}\left(q^{-\rho}\right)=q^{\kappa(\lambda) / 2} s_{\lambda}\left(q^{-\rho}\right)
$$


to rewrite (4.3) as

$$
\left.s_{\lambda}\left(q^{-\rho}\right) \Gamma_{-}\left(q^{-{ }^{\mathrm{t}} \lambda-\rho}\right)|0\rangle=\left.q^{-K / 2} \Gamma_{-}^{\prime}\left(q^{-\rho}\right) \Gamma_{+}^{\prime}\left(q^{-\rho}\right)\right|^{\mathrm{t}} \lambda\right\rangle .
$$

(4.5), in turn, is equivalent to (4.2) (with $\lambda$ being replaced by ${ }^{\mathrm{t}} \lambda$ ) as one can see from the identities

$$
\begin{aligned}
\left\langle{ }^{\mathrm{t}} \mu\left|\Gamma_{-}\left(q^{-{ }^{\mathrm{t}} \lambda-\rho}\right)\right| 0\right\rangle & =\left\langle\mu\left|\Gamma_{-}^{\prime}\left(q^{-{ }^{\mathrm{t}} \lambda-\rho}\right)\right| 0\right\rangle, \\
\left\langle{ }^{\mathrm{t}} \mu\left|\Gamma_{-}^{\prime}\left(q^{-\rho}\right) \Gamma_{+}^{\prime}\left(q^{-\rho}\right)\right|{ }^{\mathrm{t}} \lambda\right\rangle & =\left\langle\mu\left|\Gamma_{-}\left(q^{-\rho}\right) \Gamma_{+}\left(q^{-\rho}\right)\right| \lambda\right\rangle
\end{aligned}
$$

and the fact that $\langle\lambda|$ and $|\lambda\rangle$ are eigenvector of $K$ with eigenvalue $\kappa(\lambda)$. (4.2), (4.3) and (4.5) imply the relations

$$
\begin{aligned}
& s_{{ }}\left(q^{-\rho}\right)\langle 0| \Gamma_{+}^{\prime}\left(q^{-\lambda-\rho}\right)=\left\langle{ }^{t} \lambda\right| \Gamma_{-}\left(q^{-\rho}\right) \Gamma_{+}\left(q^{-\rho}\right) q^{K / 2} \\
& s_{{ }}\left(q^{-\rho}\right)\langle 0| \Gamma_{+}\left(q^{-{ }^{\mathrm{t}} \lambda-\rho}\right)=q^{\kappa(\lambda) / 2}\left\langle{ }^{\mathrm{t}} \lambda\right| \Gamma_{-}^{\prime}\left(q^{-\rho}\right) \Gamma_{+}^{\prime}\left(q^{-\rho}\right) q^{-K / 2} \text {, } \\
& s_{\lambda}\left(q^{-\rho}\right)\langle 0| \Gamma_{+}\left(q^{-{ }^{\mathrm{t}} \lambda-\rho}\right)=\left\langle{ }^{\mathrm{t}} \lambda\right| \Gamma_{-}^{\prime}\left(q^{-\rho}\right) \Gamma_{+}^{\prime}\left(q^{-\rho}\right) q^{-K / 2}
\end{aligned}
$$

in the dual Fock space as well.

Proof. The topological vertex has the fermionic expression

$$
\begin{aligned}
C_{\lambda \mu \nu} & =q^{\kappa(\mu) / 2} s_{{ }_{\nu}}\left(q^{-\rho}\right)\left\langle{ }^{\mathrm{t}} \lambda\left|\Gamma_{-}\left(q^{-\nu-\rho}\right) \Gamma_{+}\left(q^{-{ }^{\mathrm{t}} \nu-\rho}\right)\right| \mu\right\rangle \\
& =q^{\kappa(\mu) / 2} s_{{ }}\left(q^{-\rho}\right)\left\langle\lambda\left|\Gamma_{-}^{\prime}\left(q^{-\nu-\rho}\right) \Gamma_{+}^{\prime}\left(q^{-{ }^{\mathrm{t}} \nu-\rho}\right)\right|{ }^{\mathrm{t}} \mu\right\rangle .
\end{aligned}
$$

The "two-leg" case $C_{\mu \emptyset \lambda}=C_{\lambda \mu \emptyset}$ of the cyclic symmetry (2.2) $)^{4}$ thereby turns into the relation

$$
s_{{ }}\left(q^{-\rho}\right)\left\langle 0\left|\Gamma_{+}^{\prime}\left(q^{-\lambda-\rho}\right)\right| \mu\right\rangle=\left\langle{ }^{\mathrm{t}} \lambda\left|\Gamma_{-}\left(q^{-\rho}\right) \Gamma_{+}\left(q^{-\rho}\right) q^{K / 2}\right| \mu\right\rangle .
$$

among matrix elements of operators on the Fock space. Since this identity holds for any $\mu$, one obtains (4.2) in the dual form. Similarly, the symmetry relation $C_{\emptyset \mu \lambda}=C_{\mu \lambda \emptyset}$ yields the identity

$$
s_{{ }}\left(q^{-\rho}\right)\left\langle\mu\left|q^{K / 2} \Gamma_{-}\left(q^{-{ }^{t} \lambda-\rho}\right)\right| 0\right\rangle=\left\langle\mu\left|\Gamma_{-}^{\prime}\left(q^{-\rho}\right) \Gamma_{+}^{\prime}\left(q^{-\rho}\right)\right|{ }^{\mathrm{t}} \lambda\right\rangle q^{\kappa(\lambda) / 2}
$$

and this implies (4.3).

We can use the specialization

$$
\begin{aligned}
s_{\mathrm{t}_{\beta_{1}}}\left(q^{-\rho}\right)\langle 0| \Gamma_{+}^{\prime}\left(q^{-\beta_{1}-\rho}\right) & =\left\langle{ }^{\mathrm{t}} \beta_{1}\right| \Gamma_{-}\left(q^{-\rho}\right) \Gamma_{+}\left(q^{-\rho}\right) q^{K / 2}, \\
s_{{ }_{{ }}}\left(q^{-\rho}\right) \Gamma_{-}\left(q^{-{ }^{\mathrm{t}} \beta_{2}-\rho}\right)|0\rangle & =q^{\kappa\left(\beta_{2}\right) / 2} q^{-K / 2} \Gamma_{-}^{\prime}\left(q^{-\rho}\right) \Gamma_{+}^{\prime}\left(q^{-\rho}\right)\left|{ }^{\mathrm{t}} \beta_{2}\right\rangle
\end{aligned}
$$

\footnotetext{
${ }^{4}$ Zhou [30] gave a direct proof of the two-leg cyclic symmetry without relying on the crystal interpretation of Okounkov, Reshetikhin and Vafa 23. We present another direct proof in Append B that employs the same techniques as used in Section 3.
} 
of (4.2) and (4.3) to $\lambda=\beta_{1}$ and $\lambda=\beta_{2}$ to rewrite (4.1) as

$$
\begin{aligned}
Z_{\beta_{1} \beta_{2}}^{\mathrm{ctv}}= & q^{\kappa\left(\beta_{2}\right) / 2} \prod_{i, j=1}^{\infty}\left(1-Q_{1} Q_{2} q^{-\beta_{1 i}-{ }^{\mathrm{t}} \beta_{2 j}+i+j-1}\right)^{-1} \\
& \times\left\langle{ }^{\mathrm{t}} \beta_{1}\right| \Gamma_{-}\left(q^{-\rho}\right) \Gamma_{+}\left(q^{-\rho}\right) q^{K / 2}\left(-Q_{1}\right)^{L_{0}} q^{-K / 2} \Gamma_{-}^{\prime}\left(q^{-\rho}\right) \Gamma_{+}^{\prime}\left(q^{-\rho}\right)\left(-Q_{3}\right)^{L_{0}} \\
& \left.\times\left.\Gamma_{-}\left(q^{-\rho}\right) \Gamma_{+}\left(q^{-\rho}\right) q^{K / 2}\left(-Q_{2}\right)^{L_{0}} q^{-K / 2} \Gamma_{-}^{\prime}\left(q^{-\rho}\right) \Gamma_{+}^{\prime}\left(q^{-\rho}\right)\right|^{\mathrm{t}} \beta_{2}\right\rangle .
\end{aligned}
$$

Since $q^{K / 2}$ s and $q^{-K / 2}$, s in this expression cancel out as

$$
q^{K / 2}\left(-Q_{1}\right)^{L_{0}} q^{-K / 2}=\left(-Q_{1}\right)^{L_{0}}, \quad q^{K / 2}\left(-Q_{2}\right)^{L_{0}} q^{-K / 2}=\left(-Q_{2}\right)^{L_{0}},
$$

we arrive at the following final expression of $Z_{\beta_{1} \beta_{2}}^{\mathrm{ctv}}$.

Theorem 1. The open string amplitude $Z_{\beta_{1} \beta_{2}}^{\mathrm{ctv}}$ can be expressed as

$$
\begin{aligned}
Z_{\beta_{1} \beta_{2}}^{\mathrm{ctv}}= & q^{\kappa\left(\beta_{2}\right) / 2} \prod_{i, j=1}^{\infty}\left(1-Q_{1} Q_{2} q^{-\beta_{1 i}-{ }^{\mathrm{t}} \beta_{2 j}+i+j-1}\right)^{-1} \\
& \times\left\langle{ }^{\mathrm{t}} \beta_{1}\right| \Gamma_{-}\left(q^{-\rho}\right) \Gamma_{+}\left(q^{-\rho}\right)\left(-Q_{1}\right)^{L_{0}} \Gamma_{-}^{\prime}\left(q^{-\rho}\right) \Gamma_{+}^{\prime}\left(q^{-\rho}\right)\left(-Q_{3}\right)^{L_{0}} \\
& \left.\times\left.\Gamma_{-}\left(q^{-\rho}\right) \Gamma_{+}\left(q^{-\rho}\right)\left(-Q_{2}\right)^{L_{0}} \Gamma_{-}^{\prime}\left(q^{-\rho}\right) \Gamma_{+}^{\prime}\left(q^{-\rho}\right)\right|^{\mathrm{t}} \beta_{2}\right\rangle .
\end{aligned}
$$

Let us note here that the main part $\left\langle{ }^{\mathrm{t}} \beta_{1}|\cdots|{ }^{\mathrm{t}} \beta_{2}\right\rangle$ of this expression coincides with the open string amplitude of the on-strip web diagram shown in Figure 5 (see Appendix A for general formulae of amplitudes). Thus, speaking schematically, gluing the one-leg vertex (2.5) to the on-strip web diagram of Figure 4 generates another on-strip web diagram and its correction $q^{\kappa\left(\beta_{2}\right) / 2} \prod_{i, j=1}^{\infty}\left(1-Q_{1} Q_{2} \cdots\right)$. This structure of (4.10) is a key to derive $q$-difference equations for generating functions.

\section{$5 q$-difference equations for generating func- tions}

The foregoing expression (4.10) of the open string amplitudes can be used to derive $q$-difference equation for the generating functions

$$
\begin{aligned}
& \Psi(x)=\frac{1}{Z_{\emptyset \emptyset}^{\text {ctv }}} \sum_{k=0}^{\infty} Z_{\left(1^{k}\right) \emptyset}^{\mathrm{ctv}} x^{k}, \\
& \tilde{\Psi}(x)=\frac{1}{Z_{\emptyset \emptyset}^{\mathrm{ctv}}} \sum_{k=0}^{\infty} Z_{(k) \emptyset}^{\mathrm{ctv}} x^{k}
\end{aligned}
$$




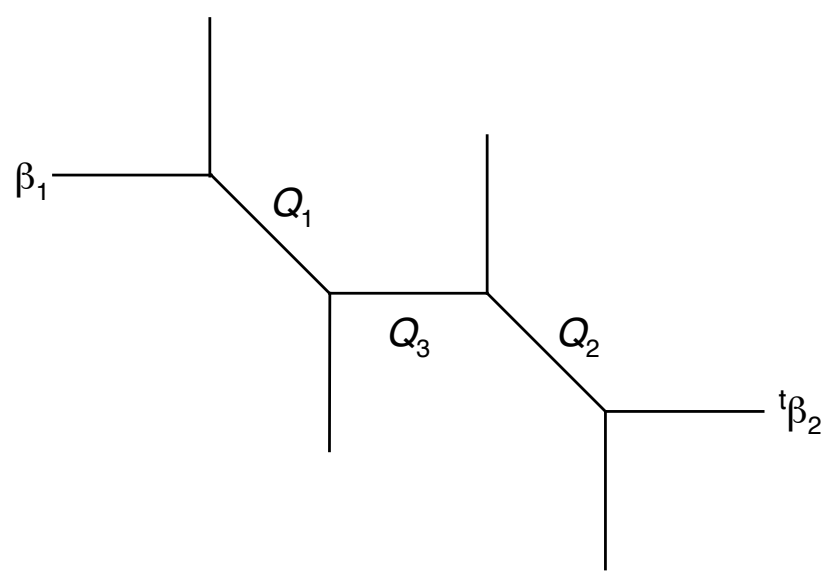

Figure 5: Web diagram emerging in (4.10)

of special subsets of the normalized amplitudes $Z_{\beta_{1} \beta_{2}}^{\text {ctv }} / Z_{\emptyset \emptyset}^{\text {ctv }}$. Note that $\left(1^{k}\right)$ ( $k$-copies of 1 ) and $(k)$ represent Young diagrams with a single column or row. These generating functions are the Baker-Akhiezer functions 5 of an integrable hierarchy, and $x$ amounts to the spectral variable therein [18]. One can derive $q$-difference equations for generating functions of $Z_{\emptyset\left(1^{k}\right)}^{\mathrm{ctv}} / Z_{\emptyset \emptyset}^{\mathrm{ctv}}$ and $Z_{\emptyset(k)}^{\text {ctv }} / Z_{\emptyset \emptyset}^{\text {ctv }}$ as well, though they become slightly more complicated because of the presence of the factor $q^{\kappa\left(\beta_{2}\right) / 2}$.

\subsection{Derivation of $q$-difference equation}

A key towards the derivation of a $q$-difference equation is to compare $\Psi(x)$ and $\tilde{\Psi}(x)$ with another pair of generating functions

$$
\begin{aligned}
& \Phi(x)=\frac{1}{Y_{\emptyset \emptyset}} \sum_{k=0}^{\infty} Y_{\left(1^{k}\right) \emptyset} x^{k}, \\
& \tilde{\Phi}(x)=\frac{1}{Y_{\emptyset \emptyset}} \sum_{k=0}^{\infty} Y_{(k) \emptyset} x^{k}
\end{aligned}
$$

obtained from the the main part

$$
\begin{aligned}
Y_{\beta_{1} \beta_{2}}= & \left\langle{ }^{\mathrm{t}} \beta_{1}\right| \Gamma_{-}\left(q^{-\rho}\right) \Gamma_{+}\left(q^{-\rho}\right)\left(-Q_{1}\right)^{L_{0}} \Gamma_{-}^{\prime}\left(q^{-\rho}\right) \Gamma_{+}^{\prime}\left(q^{-\rho}\right)\left(-Q_{3}\right)^{L_{0}} \\
& \left.\times\left.\Gamma_{-}\left(q^{-\rho}\right) \Gamma_{+}\left(q^{-\rho}\right)\left(-Q_{2}\right)^{L_{0}} \Gamma_{-}^{\prime}\left(q^{-\rho}\right) \Gamma_{+}^{\prime}\left(q^{-\rho}\right)\right|^{\mathrm{t}} \beta_{2}\right\rangle
\end{aligned}
$$

\footnotetext{
${ }^{5}$ Speaking more precisely, it is rather $\Psi(-x)$ and $\tilde{\Psi}(x)$ that literally correspond to the dual pair of Baker-Akhiezer functions. Because of this, the $q$-difference equations for $\Psi(x)$ and $\tilde{\Psi}(x)$ presented below are not fully symmetric. This is also the case for another pair $\Phi(x)$ and $\tilde{\Phi}(x)$ of generating functions introduced below.
} 
of the fermionic expression (4.10) of $Z_{\beta_{1} \beta_{2}}^{\mathrm{ctv}}$.

Let us first note the following relation between the coefficients of $\Psi(x)$ and $\Phi(x)$.

Lemma 4. The coefficients of the expansion

$$
\Psi(x)=\sum_{k=0}^{\infty} a_{k} x^{k}, \quad \Phi(x)=\sum_{k=0}^{\infty} b_{k} x^{k}, \quad a_{0}=b_{0}=1,
$$

are related as

$$
a_{k}=b_{k} \prod_{i=1}^{k}\left(1-Q_{1} Q_{2} q^{i-1}\right)^{-1} \quad \text { for } k \geq 1 .
$$

Proof. $a_{k} / b_{k}$ is given by the ratio of the values of the prefactor in (4.10) for $\beta_{1}=\left(1^{k}\right), \beta_{2}=\emptyset$ and $\beta_{1}=\beta_{2}=\emptyset$ :

$$
\begin{aligned}
\frac{a_{k}}{b_{k}} & =\prod_{i, j=1}^{\infty}\left(1-Q_{1} Q_{2} q^{-\beta_{1 i}-{ }^{\mathrm{t}} \beta_{2 j}+i+j-1}\right)^{-1} / \prod_{i, j=1}^{\infty}\left(1-Q_{1} Q_{2} q^{i+j-1}\right)^{-1} \\
& =\prod_{i=1}^{k} \prod_{j=1}^{\infty}\left(1-Q_{1} Q_{2} q^{i+j-2}\right)^{-1} / \prod_{i=1}^{k} \prod_{j=1}^{\infty}\left(1-Q_{1} Q_{2} q^{i+j-1}\right)^{-1} \\
& =\prod_{i=1}^{k}\left(1-Q_{1} Q_{2} q^{i-1}\right)^{-1} .
\end{aligned}
$$

The next step is to derive a $q$-difference equation for $\Phi(x)$.

Lemma 5. $\Phi(x)$ can be expressed in the infinite-product form

$$
\Phi(x)=\prod_{i=1}^{\infty} \frac{\left(1-Q_{1} q^{i-1 / 2} x\right)\left(1-Q_{1} Q_{2} Q_{3} q^{i-1 / 2} x\right)}{\left(1-q^{i-1 / 2} x\right)\left(1-Q_{1} Q_{3} q^{i-1 / 2} x\right)}
$$

and satisfies the q-difference equation

$$
\Phi(q x)=\frac{\left(1-q^{1 / 2} x\right)\left(1-Q_{1} Q_{3} q^{1 / 2} x\right)}{\left(1-Q_{1} q^{1 / 2} x\right)\left(1-Q_{1} Q_{2} Q_{3} q^{1 / 2} x\right)} \Phi(x) .
$$

Remark 3. The infinite product $\prod_{i=1}^{\infty}\left(1-q^{i-1 / 2} x\right)^{-1}$ is an expression of the quantum dilogarithmic function [19, 20]. Thus $\Phi(x)$ is a multiplicative combination of four quantum dilogarithmic functions. 
Proof. $Y_{\left(1^{k}\right) \emptyset}$ can be expressed as

$$
\begin{aligned}
Y_{\left(1^{k}\right) \emptyset}= & \langle(k)| \Gamma_{-}\left(q^{-\rho}\right) \Gamma_{+}\left(q^{-\rho}\right)\left(-Q_{1}\right)^{L_{0}} \Gamma_{-}^{\prime}\left(q^{-\rho}\right) \Gamma_{+}^{\prime}\left(q^{-\rho}\right)\left(-Q_{3}\right)^{L_{0}} \\
& \times \Gamma_{-}\left(q^{-\rho}\right) \Gamma_{+}\left(q^{-\rho}\right)\left(-Q_{2}\right)^{L_{0}} \Gamma_{-}^{\prime}\left(q^{-\rho}\right) \Gamma_{+}^{\prime}\left(q^{-\rho}\right)|0\rangle .
\end{aligned}
$$

By the fundamental properties

$$
\sum_{k=0}^{\infty} x^{k}\langle(k)|=\langle 0| \Gamma_{+}(x), \quad \sum_{k=0}^{\infty} x^{k}\left\langle\left(1^{k}\right)\right|=\langle 0| \Gamma_{+}^{\prime}(x),
$$

of the single-variate vertex operators [26, 27], the generating function of $Y_{\left(1^{k}\right) \emptyset}$ 's can be expressed as

$$
\begin{aligned}
\sum_{k=0}^{\infty} Y_{\left(1^{k}\right) \emptyset} x^{k}= & \langle 0| \Gamma_{+}(x) \Gamma_{-}\left(q^{-\rho}\right) \Gamma_{+}\left(q^{-\rho}\right)\left(-Q_{1}\right)^{L_{0}} \Gamma_{-}^{\prime}\left(q^{-\rho}\right) \Gamma_{+}^{\prime}\left(q^{-\rho}\right)\left(-Q_{3}\right)^{L_{0}} \\
& \times \Gamma_{-}\left(q^{-\rho}\right) \Gamma_{+}\left(q^{-\rho}\right)\left(-Q_{2}\right)^{L_{0}} \Gamma_{-}^{\prime}\left(q^{-\rho}\right) \Gamma_{+}^{\prime}\left(q^{-\rho}\right)|0\rangle .
\end{aligned}
$$

One can now use the commutation relations [26, 27]

$$
\begin{aligned}
& \Gamma_{+}(x) \Gamma_{-}(y)=(1-x y)^{-1} \Gamma_{-}(y) \Gamma_{+}(x), \\
& \Gamma_{+}^{\prime}(x) \Gamma_{-}^{\prime}(y)=(1-x y)^{-1} \Gamma_{-}^{\prime}(y) \Gamma_{+}^{\prime}(x), \\
& \Gamma_{+}(x) \Gamma_{-}^{\prime}(y)=(1+x y) \Gamma_{-}^{\prime}(y) \Gamma_{+}(x), \\
& \Gamma_{+}^{\prime}(x) \Gamma_{-}(y)=(1+x y) \Gamma_{-}(y) \Gamma_{+}^{\prime}(x)
\end{aligned}
$$

of the single-variate vertex operators to move $\Gamma_{+}(x)$ to the right until it hits $|0\rangle$ and disappears. This yields the infinite-product expression

$$
\sum_{k=0}^{\infty} Y_{\left(1^{k}\right) \emptyset} x^{k}=\prod_{i=1}^{\infty} \frac{\left(1-Q_{1} q^{i-1 / 2} x\right)\left(1-Q_{1} Q_{2} Q_{3} q^{i-1 / 2} x\right)}{\left(1-q^{i-1 / 2} x\right)\left(1-Q_{1} Q_{3} q^{i-1 / 2} x\right)} Y_{\emptyset \emptyset}
$$

of the unnormalized generating function, hence the expression (5.7) of $\Phi(x)$. The $q$-difference equation (5.8) is an immediate consequence of (5.7).

To derive a $q$-difference equation for $\Psi(x)$, let us rewrite (5.8) as

$$
\begin{aligned}
& \left(1-Q_{1}\left(1+Q_{2} Q_{3}\right) q^{1 / 2} x+Q_{1}^{2} Q_{2} Q_{3} q x^{2}\right) \Phi(q x) \\
& =\left(1-\left(1+Q_{1} Q_{3}\right) q^{1 / 2} x+Q_{1} Q_{3} q x^{2}\right) \Phi(x)
\end{aligned}
$$

and extract the coefficients of $x^{k}$. This yields the recursion relations

$$
\begin{aligned}
& q^{k} b_{k}-Q_{1}\left(1+Q_{2} Q_{3}\right) q^{1 / 2} q^{k-1} b_{k-1}+Q_{1}^{2} Q_{2} Q_{3} q q^{k-2} b_{k-2} \\
& =b_{k}-\left(1+Q_{1} Q_{3}\right) q^{1 / 2} b_{k-1}+Q_{1} Q_{3} q b_{k-2}
\end{aligned}
$$


for $b_{k}$ 's. Note that these relations hold for all $k \in \mathbb{Z}$ if $b_{k}$ 's for $k<0$ are understood to be 0 . By (5.6), these recursion relations turn into the recursion relations

$$
\begin{aligned}
& \left(1-Q_{1} Q_{2} q^{k-2}\right)\left(1-Q_{1} Q_{2} q^{k-1}\right) q^{k} a_{k} \\
& -Q_{1}\left(1+Q_{2} Q_{3}\right) q^{1 / 2}\left(1-Q_{1} Q_{2} q^{k-2}\right) q^{k-1} a_{k-1}+Q_{1}^{2} Q_{2} Q_{3} q q^{k-2} a_{k-2} \\
& =\left(1-Q_{1} Q_{2} q^{k-2}\right)\left(1-Q_{1} Q_{2} q^{k-1}\right) a_{k} \\
& \quad-\left(1+Q_{1} Q_{3}\right) q^{1 / 2}\left(1-Q_{1} Q_{2} q^{k-2}\right) a_{k-1}+Q_{1} Q_{3} q a_{k-2}
\end{aligned}
$$

for $a_{k}$ 's. Multiplying these equations by $x^{k}$ and taking the sum over $k=$ $0,1, \ldots$, we can derive a $q$-difference equation for $\Phi(x)$.

To state this result in a compact form, let us use the shift operator $q^{x \partial_{x}}$, $\partial_{x}=\partial / \partial x$, that acts on a function $f(x)$ of $x$ as

$$
q^{x \partial_{x}} f(x)=f(q x) .
$$

Theorem 2. $\Psi(x)$ satisfies the q-difference equation

$$
\begin{aligned}
& \left(1-Q_{1} Q_{2} q^{-2} q^{x \partial_{x}}\right)\left(1-Q_{1} Q_{2} q^{-1} q^{x \partial_{x}}\right) \Psi(q x) \\
& -Q_{1}\left(1+Q_{2} Q_{3}\right) q^{1 / 2} x\left(1-Q_{1} Q_{2} q^{-1} q^{x \partial_{x}}\right) \Psi(q x)+Q_{1}^{2} Q_{2} Q_{3} q x^{2} \Psi(q x) \\
& =\left(1-Q_{1} Q_{2} q^{-2} q^{x \partial_{x}}\right)\left(1-Q_{1} Q_{2} q^{-1} q^{x \partial_{x}}\right) \Psi(x) \\
& \quad-\left(1+Q_{1} Q_{3}\right) q^{1 / 2} x\left(1-Q_{1} Q_{2} q^{-1} q^{x \partial_{x}}\right) \Psi(x)+Q_{1} Q_{3} q x^{2} \Psi(x) .
\end{aligned}
$$

A $q$-difference equation for $\tilde{\Psi}(x)$ can be derived in the same way from the $q$-difference equation

$$
\tilde{\Phi}(q x)=\frac{\left(1+Q_{1} q^{1 / 2} x\right)\left(1+Q_{1} Q_{2} Q_{3} q^{1 / 2} x\right)}{\left(1+q^{1 / 2} x\right)\left(1+Q_{1} Q_{3} q^{1 / 2} x\right)} \tilde{\Phi}(x)
$$

for $\tilde{\Phi}(x)$ and the relation

$$
\tilde{a}_{k}=\tilde{b}_{k} \prod_{i=1}^{k}\left(1-Q_{1} Q_{2} q^{1-i}\right)^{-1} \quad \text { for } k \geq 1
$$

between the coefficients of the expansion

$$
\tilde{\Psi}(x)=\sum_{k=0}^{\infty} \tilde{a}_{k} x^{k}, \quad \tilde{\Phi}(x)=\sum_{k=0}^{\infty} \tilde{b}_{k} x^{k}, \quad \tilde{a}_{0}=\tilde{b}_{0}=1,
$$

of $\tilde{\Psi}(x)$ and $\tilde{\Phi}(x)$. We omit the detail of calculation and show the result: 
Theorem 3. $\tilde{\Psi}(x)$ satisfies the q-difference equation

$$
\begin{aligned}
& \left(1-Q_{1} Q_{2} q^{2} q^{-x \partial_{x}}\right)\left(1-Q_{1} Q_{2} q q^{-x \partial_{x}}\right) \tilde{\Psi}\left(q^{-1} x\right) \\
& +Q_{1}\left(1+Q_{2} Q_{3}\right) q^{-1 / 2} x\left(1-Q_{1} Q_{2} q q^{-x \partial_{x}}\right) \tilde{\Psi}\left(q^{-1} x\right)+Q_{1}^{2} Q_{2} Q_{3} q^{-1} x^{2} \tilde{\Psi}\left(q^{-1} x\right) \\
& =\left(1-Q_{1} Q_{2} q^{2} q^{-x \partial_{x}}\right)\left(1-Q_{1} Q_{2} q q^{-x \partial_{x}}\right) \tilde{\Psi}(x) \\
& \quad+\left(1+Q_{1} Q_{3}\right) q^{-1 / 2} x\left(1-Q_{1} Q_{2} q q^{-x \partial_{x}}\right) \tilde{\Psi}(x)+Q_{1} Q_{3} q^{-1} x^{2} \tilde{\Psi}(x) .
\end{aligned}
$$

Remark 4. Both sides of (5.13) and (5.16) can be rewritten as

$$
\begin{aligned}
& \left(1-Q_{1} Q_{2} q^{-2} q^{x \partial_{x}}-Q_{1} q^{1 / 2} x\right)\left(1-Q_{1} Q_{2} q^{-1} q^{x \partial_{x}}-Q_{1} Q_{2} Q_{3} q^{1 / 2} x\right) \Psi(q x) \\
& =\left(1-Q_{1} Q_{2} q^{-2} q^{x \partial_{x}}-q^{1 / 2} x\right)\left(1-Q_{1} Q_{2} q^{-1} q^{x \partial_{x}}-Q_{1} Q_{3} q^{1 / 2} x\right) \Psi(x)
\end{aligned}
$$

and

$$
\begin{aligned}
& \left(1-Q_{1} Q_{2} q^{2} q^{-x \partial_{x}}+Q_{1} q^{-1 / 2} x\right)\left(1-Q_{1} Q_{2} q q^{-x \partial_{x}}+Q_{1} Q_{2} Q_{3} q^{-1 / 2} x\right) \tilde{\Psi}\left(q^{-1} x\right) \\
& =\left(1-Q_{1} Q_{2} q^{2} q^{-x \partial_{x}}+q^{-1 / 2} x\right)\left(1-Q_{1} Q_{2} q q^{-x \partial_{x}}+Q_{1} Q_{3} q^{-1 / 2} x\right) \tilde{\Psi}(x)
\end{aligned}
$$

This expression corresponds to writing $q$-difference equations for $\Phi(x)$ and $\tilde{\Phi}(x)$ as

$$
\left(1-Q_{1} q^{1 / 2} x\right)\left(1-Q_{1} Q_{2} Q_{3} q^{1 / 2} x\right) \Phi(q x)=\left(1-q^{1 / 2} x\right)\left(1-Q_{1} Q_{3} q^{1 / 2} x\right) \Phi(x)
$$

and

$$
\left(1+Q_{1} q^{-1 / 2} x\right)\left(1+Q_{1} Q_{2} Q_{3} q^{-1 / 2} x\right) \tilde{\Phi}\left(q^{-1} x\right)=\left(1+q^{-1 / 2} x\right)\left(1+Q_{1} Q_{3} q^{-1 / 2} x\right) \tilde{\Phi}(x) .
$$

These $q$-difference equations are transformed to the foregoing ones for $\Psi(x)$ and $\tilde{\Psi}(x)$ by the transformation (5.6) and (5.15) of the coefficients.

\subsection{Structure of $q$-difference operators}

Let us rewrite (5.13) as

$$
H\left(x, q^{x \partial_{x}}\right) \Psi(x)=0
$$

and examine the structure of the $q$-difference operator $H$. This operator reads

$$
\begin{aligned}
H\left(x, q^{x \partial_{x}}\right)= & \left(1-Q_{1} Q_{2} q^{-2} q^{x \partial_{x}}\right)\left(1-Q_{1} Q_{2} q^{-1} q^{x \partial_{x}}\right) \\
& -\left(1+Q_{1} Q_{3}\right) q^{1 / 2} x\left(1-Q_{1} Q_{2} q^{-1} q^{x \partial_{x}}\right)+Q_{1} Q_{3} q x^{2} \\
& -\left(1-Q_{1} Q_{2} q^{-2} q^{x \partial_{x}}\right)\left(1-Q_{1} Q_{2} q^{-1} q^{x \partial_{x}}\right) q^{x \partial_{x}} \\
& +Q_{1}\left(1+Q_{2} Q_{3}\right) q^{1 / 2} x\left(1-Q_{1} Q_{2} q^{-1} q^{x \partial_{x}}\right) q^{x \partial_{x}}-Q_{1}^{2} Q_{2} Q_{3} q x^{2} q^{x \partial_{x}}
\end{aligned}
$$


Remarkably, $H\left(x, q^{x \partial_{x}}\right)$ can be factorized as

$$
H\left(x, q^{x \partial_{x}}\right)=\left(1-Q_{1} Q_{2} q^{-2} q^{x \partial_{x}}\right) K\left(x, q^{x \partial_{x}}\right),
$$

where

$$
\begin{aligned}
K\left(x, q^{x \partial_{x}}\right)= & \left(1-Q_{1} Q_{2} q^{-1} q^{x \partial_{x}}\right)\left(1-q^{x \partial_{x}}\right)-\left(1+Q_{1} Q_{3}\right) q^{1 / 2} x \\
& +Q_{1}\left(1+Q_{2} Q_{3}\right) q^{1 / 2} x q^{x \partial_{x}}+Q_{1} Q_{3} q x^{2}
\end{aligned}
$$

This is also the case for the $q$-difference equation (5.16) for $\tilde{\Psi}(x)$. The $q$-difference operator $\tilde{H}\left(x, q^{x \partial_{x}}\right)$ in the expression

$$
\tilde{H}\left(x, q^{x \partial_{x}}\right) \tilde{\Psi}(x)=0
$$

of (5.16) reads

$$
\begin{aligned}
\tilde{H}\left(x, q^{x \partial_{x}}\right)= & \left(1-Q_{1} Q_{2} q^{2} q^{-x \partial_{x}}\right)\left(1-Q_{1} Q_{2} q q^{-x \partial_{x}}\right) \\
& +\left(1+Q_{1} Q_{3}\right) q^{1 / 2} x\left(1-Q_{1} Q_{2} q q^{-x \partial_{x}}\right)+Q_{1} Q_{3} q x^{2} \\
& -\left(1-Q_{1} Q_{2} q^{2} q^{-x \partial_{x}}\right)\left(1-Q_{1} Q_{2} q q^{-x \partial_{x}}\right) q^{-x \partial_{x}} \\
& -Q_{1}\left(1+Q_{2} Q_{3}\right) q^{1 / 2} x\left(1-Q_{1} Q_{2} q q^{-x \partial_{x}}\right) q^{-x \partial_{x}}-Q_{1}^{2} Q_{2} Q_{3} q x^{2} q^{-x \partial_{x}}
\end{aligned}
$$

This operator can be factorized as

$$
\tilde{H}\left(x, q^{x \partial_{x}}\right)=\left(1-Q_{1} Q_{2} q^{2} q^{-x \partial_{x}}\right) \tilde{K}\left(x, q^{x \partial_{x}}\right),
$$

where

$$
\begin{aligned}
\tilde{K}\left(x, q^{x \partial_{x}}\right)= & \left(1-Q_{1} Q_{2} q q^{-x \partial_{x}}\right)\left(1-q^{-x \partial_{x}}\right)+\left(1+Q_{1} Q_{3}\right) q^{1 / 2} x \\
& -Q_{1}\left(1+Q_{2} Q_{3}\right) q^{1 / 2} x q^{-x \partial_{x}}+Q_{1} Q_{3} q x^{2}
\end{aligned}
$$

Let us note here that the action of $1-Q_{1} Q_{2} q^{-2} q^{x \partial_{x}}$ and $1-Q_{1} Q_{2} q^{2} q^{-x \partial_{x}}$ on the space of power series of $x$ is invertible as far as $Q_{1}$ and $Q_{2}$ take generic values, i.e., apart from the exceptional cases where $Q_{1} Q_{2}=q^{n}, n \in \mathbb{Z}$. Therefore these factors can be removed from the $q$-difference equations (5.21) and (5.25). Actually, this genericity is implicitly assumed in the transformations (5.6) and (5.15) of these generating functions. Thus we find the following refinement of Theorems 2 and 3 .

Theorem 4. For generic values of $Q_{1}$ and $Q_{2}$, the q-difference equations (5.13) and (5.16) can be reduced to

$$
K\left(x, q^{x \partial_{x}}\right) \Psi(x)=0, \quad \tilde{K}\left(x, q^{x \partial_{x}}\right) \tilde{\Psi}(x)=0 .
$$



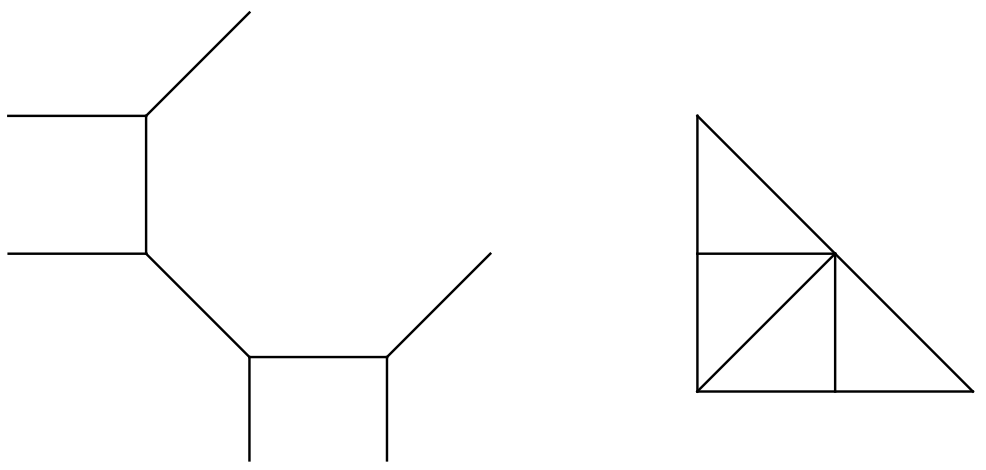

Figure 6: Web and toric diagrams after flop

This result fits well into the perspectives of mirror geometry of topological string theory on non-compact toric Calabi-Yau threefolds [6, 7]. $\Psi(x)$ and $\tilde{\Psi}(x)$ may be thought of as wave functions of a probe D-brane. In this interpretation, a $q$-difference equation satisfied by these functions defines a quantum mirror curve. The q-difference equations (5.29) indeed have such a characteristic. In the classical limit as $q \rightarrow 1$, the non-commutative polynomials $K\left(x, q^{x \partial_{x}}\right)$ and $\tilde{K}\left(x, q^{-x \partial_{x}}\right)$ turn into the ordinary polynomials

$$
\begin{aligned}
K_{\mathrm{cl}}(x, y)= & \left(1-Q_{1} Q_{2} y\right)(1-y)-\left(1+Q_{1} Q_{3}\right) x \\
& +Q_{1}\left(1+Q_{2} Q_{3}\right) x y+Q_{1} Q_{3} x^{2}
\end{aligned}
$$

in $(x, y)$ and

$$
\begin{aligned}
\tilde{K}_{\mathrm{cl}}(x, y)= & \left(1-Q_{1} Q_{2} y^{-1}\right)\left(1-y^{-1}\right)+\left(1+Q_{1} Q_{3}\right) x \\
& -Q_{1}\left(1+Q_{2} Q_{3}\right) x y^{-1}+Q_{1} Q_{3} x^{2}
\end{aligned}
$$

in $\left(x, y^{-1}\right)$. As expected from the perspectives of mirror geometry, the Newton polygons of these polynomials have the same shape as the toric diagram in Figure 1.

\section{Flop transition}

Let us examine the flop transition from Figure 1 to Figure 6. After this move, the previous setup for defining the amplitude $Z_{\beta_{1} \beta_{2}}^{\text {ctv }}$ turns into the setup shown in Figure 7. Note that the Kähler parameters after the flop transition are denoted by $P_{1}, P_{2}, P_{3}$; they are expected to be related to the Kähler parameters $Q_{1}, Q_{2}, Q_{3}$ before the transition by birational transformations.

Our method for calculating $Z_{\beta_{1} \beta_{2}}^{\text {ctv }}$ can be extended to the amplitude $\hat{Z}_{\beta_{1} \beta_{2}}^{\text {ctv }}$ of Figure 7 as follows. 


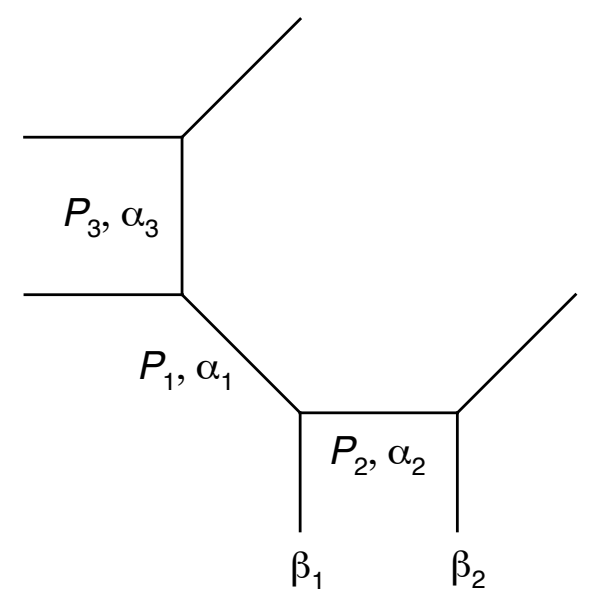

Figure 7: Setup for open string amplitude $\hat{Z}_{\beta_{1} \beta_{2}}^{\mathrm{ctv}}$

The sum over $\alpha_{1}, \alpha_{2}, \alpha_{3} \in \mathcal{P}$ can be decomposed to a partial sum $\hat{Z}_{\beta_{1} \beta_{2}}^{\alpha_{3}}$ with respect to $\alpha_{1}, \alpha_{2}$ at the first stage and a sum with respect to $\alpha_{3}$ at the next stage as

$$
\hat{Z}_{\beta_{1} \beta_{2}}^{\mathrm{ctv}}=\sum_{\alpha_{3} \in \mathcal{P}} \hat{Z}_{\beta_{1} \beta_{2} \mid \alpha_{3}}\left(-P_{3}\right)^{\left|\alpha_{3}\right|}(-1)^{\left|\alpha_{3}\right|} q^{-\kappa\left(\alpha_{3}\right) / 2} C_{\mathrm{t}_{\alpha_{3}} \emptyset \emptyset}
$$

The extra factor $(-1)^{\left|\alpha_{3}\right|} q^{-\kappa\left(\alpha_{3}\right) / 2}$ is inserted by the gluing rule. The framing number (2.3) along the internal line carrying $\alpha_{3}$ is equal to 1.

The partial sum $\hat{Z}_{\beta_{1} \beta_{2} \mid \alpha_{3}}$ is an open string amplitude of the double- $\mathbb{P}^{1}$ diagram shown in Figure 8. Since this is an on-strip diagram, the amplitude can be calculated explicitly as

$$
\begin{aligned}
\hat{Z}_{\beta_{1} \beta_{2} \mid \alpha_{3}}= & s{ }_{\mathrm{t}_{1}}\left(q^{-\rho}\right) s \mathrm{t}_{\beta_{2}}\left(q^{-\rho}\right) s{ }^{{ }} \alpha_{3}\left(q^{-\rho}\right) \prod_{i, j=1}^{\infty}\left(1-P_{2} q^{-\beta_{1 i}-{ }^{\mathrm{t}} \beta_{2 j}+i+j-1}\right)^{-1} \\
& \times \prod_{i, j=1}^{\infty}\left(1-P_{1} q^{-{ }^{\mathrm{t}} \alpha_{3 i}-{ }^{\mathrm{t}} \beta_{1 j}+i+j-1}\right) \prod_{i, j=1}^{\infty}\left(1-P_{1} P_{2} q^{-{ }^{\mathrm{t}} \alpha_{3 i}-{ }^{\mathrm{t}} \beta_{2 j}+i+j-1}\right) .
\end{aligned}
$$

This amplitude is related to its counterpart $Z_{\beta_{1} \beta_{2} \mid \alpha_{3}}$ by the same flop operation as the move from Figure 1 to Figure 6. One see form (2.6) and (6.2) that $\hat{Z}_{\beta_{1} \beta_{2} \mid \alpha_{3}}$ is almost identical to $Z_{\beta_{1} \beta_{2} \mid \alpha_{3}}$ if the Kähler parameters are related as

$$
P_{1} P_{2}=Q_{2}, \quad P_{2}=Q_{1} Q_{2} .
$$

The only discrepancy lies in the infinite products $\prod_{i, j=1}^{\infty}\left(1-Q_{1} q^{\cdots}\right)$ in $(2.6)$ and $\prod_{i, j=1}^{\infty}\left(1-P_{1} q^{\cdots}\right)$ in (․․2). 

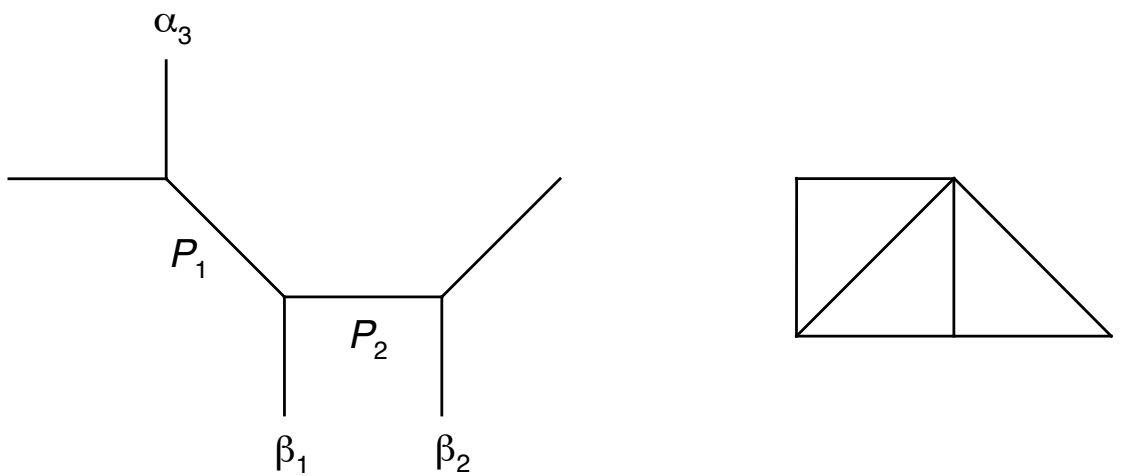

Figure 8: Double- $\mathbb{P}^{1}$ diagram defining $\hat{Z}_{\beta_{1} \beta_{2} \mid \alpha_{3}}$

Substituting (6.2) and (2.5) in (6.1), we obtain the following expression of $\hat{Z}_{\beta_{1} \beta_{2}}^{\mathrm{ctv}}$ :

$$
\begin{aligned}
\hat{Z}_{\beta_{1} \beta_{2}}^{\mathrm{ctv}}= & s_{{ }_{\beta_{1}}}\left(q^{-\rho}\right) s_{{ }_{\beta_{2}}}\left(q^{-\rho}\right) \prod_{i, j=1}^{\infty}\left(1-P_{2} q^{-\beta_{1 i}-{ }^{\mathrm{t}} \beta_{2 j}+i+j-1}\right)^{-1} \\
& \times \sum_{\alpha_{3} \in \mathcal{P}} s_{\alpha_{3}}\left(q^{-\rho}\right)^{2} P_{3}^{\left|\alpha_{3}\right|} \prod_{i, j=1}^{\infty}\left(1-P_{1} q^{-{ }^{\mathrm{t}} \alpha_{3 i}-{ }^{\mathrm{t}} \beta_{1 j}+i+j-1}\right) \\
& \times \prod_{i, j=1}^{\infty}\left(1-P_{1} P_{2} q^{-{ }^{\mathrm{t}} \alpha_{3 i}-{ }^{\mathrm{t}} \beta_{2 j}+i+j-1}\right)
\end{aligned}
$$

Note that we have used the identity (4.4) as well to rewrite the first part of the summand as

$$
s^{\mathrm{t}_{\alpha_{3}}}\left(q^{-\rho}\right) s_{\alpha_{3}}\left(q^{-\rho}\right) q^{-\kappa\left(\alpha_{3}\right) / 2}=s_{\alpha_{3}}\left(q^{-\rho}\right)^{2} .
$$

Thus, in contrast with (2.7), the sum in this case resembles the partition function of the ordinary melting crystal model [9, 10] for which the main part of the Boltzmann weight is $s_{\alpha_{3}}\left(q^{-\rho}\right)^{2}$ rather than $s_{{ }^{t} \alpha_{3}}\left(q^{-\rho}\right) s_{\alpha_{3}}\left(q^{-\rho}\right)$.

The sum in (6.4) can be calculated in more or less the same way as the case of (2.7). Let us show the final result only.

Theorem 5. The open string amplitude $\hat{Z}_{\beta_{1} \beta_{2}}^{\mathrm{ctv}}$ can be expressed as

$$
\begin{aligned}
\hat{Z}_{\beta_{1} \beta_{2}}^{\mathrm{ctv}}= & q^{\kappa\left(\beta_{1}\right) / 2+\kappa\left(\beta_{2}\right) / 2} \prod_{i, j=1}^{\infty}\left(1-P_{2} q^{-\beta_{1 i}-{ }^{\mathrm{t}} \beta_{2 j}+i+j-1}\right)^{-1} \\
& \times\left\langle{ }^{\mathrm{t}} \beta_{1}\right| \Gamma_{-}^{\prime}\left(q^{-\rho}\right) \Gamma_{+}^{\prime}\left(q^{-\rho}\right)\left(-P_{1}\right)^{L_{0}} \Gamma_{-}\left(q^{-\rho}\right) \Gamma_{+}\left(q^{-\rho}\right) P_{3}^{L_{0}} \\
& \left.\times\left.\Gamma_{-}\left(q^{-\rho}\right) \Gamma_{+}\left(q^{-\rho}\right)\left(-P_{1} P_{2}\right)^{L_{0}} \Gamma_{-}^{\prime}\left(q^{-\rho}\right) \Gamma_{+}^{\prime}\left(q^{-\rho}\right)\right|^{\mathrm{t}} \beta_{2}\right\rangle .
\end{aligned}
$$




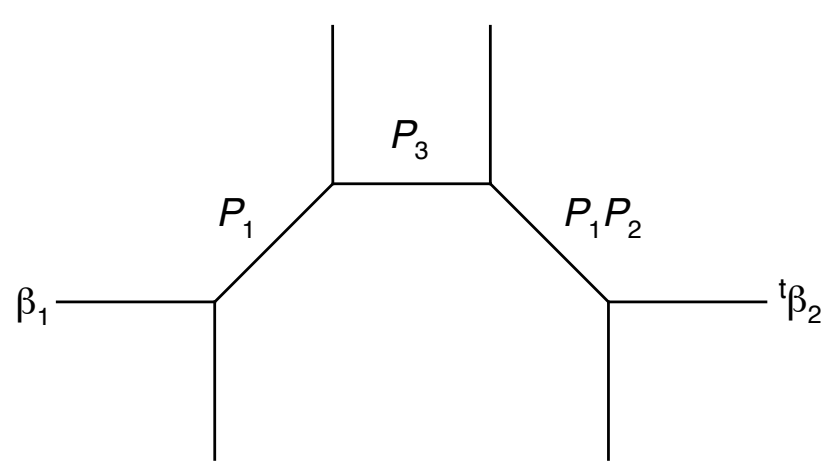

Figure 9: Web diagram emerging in (6.5)

The main part $\left\langle{ }^{\mathrm{t}} \beta_{1}|\cdots|{ }^{\mathrm{t}} \beta_{2}\right\rangle$ of this expression is essentially the open string amplitude of the web diagram shown in Figure 9. This web diagram can be derived the web diagram of Figure 5 by the same flop operation as the move from Figure 1 to Figure 6 .

To see how this part is related to the main part of (4.10), let us use the commutation relations (5.10) to exchange the order of the first four vertex operators therein as

$$
\begin{aligned}
\left\langle{ }^{\mathrm{t}} \beta_{1}\right| \Gamma_{-}^{\prime}\left(q^{-\rho}\right) \Gamma_{+}^{\prime}\left(q^{-\rho}\right)\left(-P_{1}\right)^{L_{0}} \Gamma_{-}\left(q^{-\rho}\right) \Gamma_{+}\left(q^{-\rho}\right) P_{3}^{L_{0}} \\
=\left\langle{ }^{\mathrm{t}} \beta_{1}\right|\left(-P_{1}\right)^{L_{0}} \Gamma_{-}^{\prime}\left(-P_{1}^{-1} q^{-\rho}\right) \Gamma_{+}^{\prime}\left(-P_{1} q^{-\rho}\right) \Gamma_{-}\left(q^{-\rho}\right) \Gamma_{+}\left(q^{-\rho}\right) P_{3}^{L_{0}} \\
=\left(-P_{1}\right){ }^{\left|\beta_{1}\right|} \prod_{i, j=1}^{\infty}\left(1-P_{1} q^{i+j-1}\right)\left(1-P_{1}^{-1} q^{i+j-1}\right)^{-1} \\
\quad \times\left\langle{ }^{\mathrm{t}} \beta_{1}\right| \Gamma_{-}\left(q^{-\rho}\right) \Gamma_{+}\left(q^{-\rho}\right) \Gamma_{-}^{\prime}\left(-P_{1}^{-1} q^{-\rho}\right) \Gamma_{+}^{\prime}\left(-P_{1} q^{-\rho}\right) P_{3}^{L_{0}} \\
=\left(-P_{1}\right)^{\left|\beta_{1}\right|} \prod_{i, j=1}^{\infty}\left(1-P_{1} q^{i+j-1}\right)\left(1-P_{1}^{-1} q^{i+j-1}\right)^{-1} \\
\quad \times\left\langle{ }^{\mathrm{t}} \beta_{1}\right| \Gamma_{-}\left(q^{-\rho}\right) \Gamma_{+}\left(q^{-\rho}\right)\left(-P_{1}^{-1}\right)^{L_{0}} \Gamma_{-}^{\prime}\left(q^{-\rho}\right) \Gamma_{+}^{\prime}\left(q^{-\rho}\right)\left(-P_{1} P_{3}\right)^{L_{0}} .
\end{aligned}
$$

This shows that if the two sets of Kähler parameters are matched as

$$
P_{1}^{-1}=Q_{1}, \quad P_{1} P_{3}=Q_{3}, \quad P_{1} P_{2}=Q_{2},
$$

$\hat{Z}_{\beta_{1} \beta_{2}}^{\mathrm{ctv}}$ and $Z_{\beta_{1} \beta_{2}}^{\mathrm{ctv}}$ are related as

$$
\hat{Z}_{\beta_{1} \beta_{2}}^{\mathrm{ctv}}=q^{\kappa\left(\beta_{1}\right) / 2}\left(-P_{1}\right)^{\left|\beta_{1}\right|} \prod_{i, j=1}^{\infty}\left(1-P_{1} q^{i+j-1}\right)\left(1-P_{1}^{-1} q^{i+j-1}\right)^{-1} \cdot Z_{\beta_{1} \beta_{2}}^{\mathrm{ctv}} .
$$

Note that (6.6) is consistent with (6.3) . These matching rules of parameters agree with the known result for the partition functions [2, 5, 31]. 
Remark 5. It is instructive to examine a different cut-and-glue procedure in this case. Let us try to cut the middle internal line (to which $Q_{1}$ and $\alpha_{1}$ are assigned) of the web diagram (see Figure 7). The cutting procedure yields two subdiagrams of the on-strip type. They are glued together with the edge weight $\left(-P_{1}\right)^{\left|\alpha_{1}\right|}$. Note that the framing number in this case is equal to 0 . Thus the total amplitude can be expressed as

$$
\hat{Z}_{\beta_{1} \beta_{2}}^{\mathrm{ctv}}=\sum_{\alpha_{1} \in \mathcal{P}} \hat{Z}_{\alpha_{1}}^{\prime}\left(-P_{1}\right)^{\left|\alpha_{1}\right|} \hat{Z}_{\alpha_{1} \mid \beta_{1} \beta_{2}}^{\prime \prime}
$$

where $\hat{Z}_{\alpha_{1}}^{\prime}$ and $\hat{Z}_{\alpha_{1} \mid \beta_{1} \beta_{2}}^{\prime \prime}$ are contributions of the two on-strip subdiagrams, i.e.,

$$
\hat{Z}_{\alpha_{1}}^{\prime}=\left\langle 0\left|\Gamma_{-}^{\prime}\left(q^{-\rho}\right) \Gamma_{+}^{\prime}\left(q^{-\rho}\right) P_{3}^{L_{0}} \Gamma_{-}^{\prime}\left(q^{-\rho}\right) \Gamma_{+}^{\prime}\left(q^{-\rho}\right)\right| \alpha_{1}\right\rangle
$$

and

$$
\begin{aligned}
\hat{Z}_{\alpha_{1} \mid \beta_{1} \beta_{2}}^{\prime \prime}= & q^{-\kappa\left(\alpha_{1}\right) / 2} s_{\mathrm{t}_{\beta_{1}}}\left(q^{-\rho}\right) s \mathrm{~s}_{\beta_{2}}\left(q^{-\rho}\right) \\
& \times\left\langle\alpha_{1}\left|\Gamma_{-}^{\prime}\left(q^{-{ }^{\mathrm{t}} \beta_{1}-\rho}\right) \Gamma_{+}^{\prime}\left(q^{-\beta_{1}-\rho}\right) P_{2}^{L_{0}} \Gamma_{-}^{\prime}\left(q^{-{ }^{\mathrm{t}} \beta_{2}-\rho}\right) \Gamma_{+}^{\prime}\left(q^{-\beta_{2}-\rho}\right)\right| 0\right\rangle .
\end{aligned}
$$

Plugging these expressions into (6.8) leads to yet another fermionic expression of $\hat{Z}_{\beta_{1} \beta_{2}}^{\mathrm{ctv}}$ :

$$
\begin{aligned}
\hat{Z}_{\beta_{1} \beta_{2}}^{\mathrm{ctv}}= & s_{\mathrm{t}_{\beta_{1}}}\left(q^{-\rho}\right) s_{\mathrm{t}_{\beta_{2}}}\left(q^{-\rho}\right) \\
& \times\langle 0| \Gamma_{-}^{\prime}\left(q^{-\rho}\right) \Gamma_{+}^{\prime}\left(q^{-\rho}\right) P_{3}^{L_{0}} \Gamma_{-}^{\prime}\left(q^{-\rho}\right) \Gamma_{+}^{\prime}\left(q^{-\rho}\right) q^{-K / 2}\left(-P_{1}\right)^{L_{0}} \\
& \times \Gamma_{-}^{\prime}\left(q^{-{ }^{\mathrm{t}} \beta_{1}-\rho}\right) \Gamma_{+}^{\prime}\left(q^{-\beta_{1}-\rho}\right) P_{2}^{L_{0}} \Gamma_{-}^{\prime}\left(q^{-{ }^{\mathrm{t}} \beta_{2}-\rho}\right) \Gamma_{+}^{\prime}\left(q^{-\beta_{2}-\rho}\right)|0\rangle .
\end{aligned}
$$

This expression looks very similar to an on-strip amplitude. The operator product in this expression, however, contains the operator $q^{-K / 2}$ that does not appear in on-strip amplitudes. Because of this operator, one cannot calculate this expression directly. In contrast, if one applies the cut-andglue procedure to an on-strip amplitude, operators of the form $q^{ \pm K / 2}$ do not appear or cancel out in the outcome of calculation 6 . This cancellation mechanism is a consequence of the linear shape of the on-strip diagram. In this respect, the web diagram of Figure 6 is a chain of on-strip diagrams, and its web diagram is bent to ninety degrees in the middle. It is this bend that generates the operator $q^{-K / 2}$. Actually, the present case is special in the sense that this difficulty can be circumvented by the foregoing different cut-and-glue description]. In a general case, such an escape route is not prepared.

\footnotetext{
${ }^{6}$ This is a key to prove the fermionic formula (A.1) of on-strip amplitudes by induction.

7 One can also convert (6.9) to a more tractable form with the aid of techniques used in Sections 3 and 4 . This eventually leads to the same result as presented therein.
} 


\section{Conclusion}

Let us summarize what we have done in this paper.

Calculation of open string amplitudes We reformulated the open string amplitude $Z_{\beta_{1} \beta_{2}}^{\mathrm{ctv}}$ of Figure 2 in the partially summed form (2.4), and derived the reduced expression (2.7). The main part of (2.7) turns out to be similar to the partition function of the modified melting crystal model. Firstly, the main part $s{ }^{{ }} \alpha_{3}\left(q^{-\rho}\right) s_{\alpha_{3}}\left(q^{-\rho}\right)$ of the summand is exactly the same. Secondly, the other part can be described by matrix elements of the diagonal operators $V_{0}^{( \pm)}$in the quantum torus algebra. This is also a characteristic of the external potentials in the melting crystal models. We could thereby apply the method for the melting crystal models to derive the fermionic expression (4.1) of $Z_{\beta_{1} \beta_{2}}^{\mathrm{ctv}}$. This expression was further converted to the final expression (4.10) of $Z_{\beta_{1} \beta_{2}}^{\text {ctv }}$, which is a product of a simple prefactor and the open string amplitude $Y_{\beta_{1} \beta_{2}}$ of a new on-strip diagram.

Derivation of $q$-difference equations We derived $q$-difference equations for the generating functions $\Psi(x), \tilde{\Psi}(x)$ of the normalized amplitudes $Z_{\beta_{1} \beta_{2}}^{\text {ctv }} / Z_{\emptyset \emptyset}^{\text {ctv }}$ specialized to $\beta_{1}=\left(1^{k}\right),(k), k=0,1,2, \ldots$, and $\beta_{2}=\emptyset$. The derivation makes full use of the factorized form of (4.10). Namely, we first derived the $q$-difference equations (5.8) and (5.14) for the generating functions $\Phi(x), \tilde{\Phi}(x)$ obtained from $Y_{\beta_{1} \beta_{2}} / Y_{\emptyset \emptyset}$. These equations are transformed to the $q$-difference equations (5.13) and (5.16) for $\Psi(x), \tilde{\Psi}(x)$. This is the place where the prefactor of $Y_{\beta_{1} \beta_{2}}$ in (4.10) plays a role. We examined the structure of these $q$-difference equations and found that they can be reduced to the simpler equations (5.29). It is these reduced equations that should be interpreted as the defining equation of a quantum mirror curve.

Flop transition We considered the flop transition from Figure 1 to Figure 6. The open string amplitude $\hat{Z}_{\beta_{1} \beta_{2}}^{\mathrm{ctv}}$ after the transition can be calculated in much the same way as in the case of $Z_{\beta_{1} \beta_{2}}^{\text {ctv }}$. We confirmed that $\hat{Z}_{\beta_{1} \beta_{2}}^{\text {ctv }}$ can be matched to the amplitude $Z_{\beta_{1} \beta_{2}}^{\mathrm{ctv}}$ by the birational transformations (6.6) of the Kähler parameters.

On the other hand, we have been unable to derive $q$-difference equations in other configurations of partitions on the external lines of the web diagram (except for those that can be derived from the setup of Section 2 by symmetries or specializations of the amplitude). A major obstacle is the emergence of $q^{ \pm K / 2}$ 's that do not cancel out in a fermionic expression of the amplitude as 


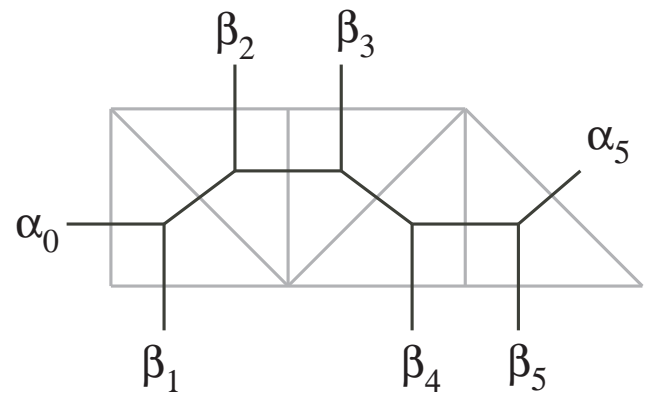

Figure 10: Web diagram on triangulated strip

opposed to the case of (4.9). Because of this obstacles, the fermionic expression in such a case cannot be converted to a form from which a $q$-difference equation can be read out.

We have encountered the same difficulty in an attempt to extend our results to more general tree-like web diagrams studied by Karp, Liu and Mariño [4. Our attempt has been unsuccessful not only for open string amplitudes, but also for the closed string partition function. We believe that this difficulty is of technical nature and can be overcome by a new computational idea.

\section{Acknowledgements}

The authors are grateful to Motohico Mulase for valuable comments. This work is partly supported by JSPS Kakenhi Grant No. 24540223, No. 25400111 and No. 15K04912.

\section{A Amplitudes of on-strip geometry}

The toric diagram of on-strip geometry is a triangulation of the strip of height 1 to triangles of area 1/2 (see Figure 10). The associated web diagram is a connected acyclic graph. If the toric graph comprises $N$ triangles, the web diagram has $N$ vertices, $N-1$ internal lines and $N+2$ external lines. The $N$ external lines other than the leftmost and rightmost ones are vertical. For brevity, the external lines are also referred to as "legs".

We assign the Kähler parameters $Q_{1}, \ldots, Q_{N-1}$ to the internal lines, the partitions $\beta_{1}, \ldots, \beta_{N}$ to the vertical external lines, and the partitions $\alpha_{0}, \alpha_{N}$

\footnotetext{
${ }^{8}$ The situation presented in Remark 5 is similar, but the difficulty in that case can be circumvented.
} 
to the leftmost and rightmost external lines. Let $Z_{\beta_{1} \cdots \beta_{N}}^{\alpha_{0} \alpha_{N}}$ denote the open string amplitude in this setup. This amplitude is defined as a sum of the product of vertex and edge weights with respect to the partitions $\alpha_{1}, \ldots, \alpha_{N-1}$ on the internal lines. In the case of $\alpha_{0}=\alpha_{N}=\emptyset$, Iqbal and Kashani-Poor [2] calculated this sum in a closed form by skillful use of the Cauchy identities for skew Schur functions. Their result can be reformulated, without restriction to $\alpha_{0}=\alpha_{N}=\emptyset$, in the language of fermions [32, 33, 34].

Following the notations of Nagao [33] and Sułkowski [34], let us define the sign (or type) $\sigma_{n}= \pm 1$ of the $n$-th vertex as:

(i) $\sigma_{n}=+1$ if the vertical leg points up,

(ii) $\sigma_{n}=-1$ if the vertical leg points down.

For example, in the case of the web diagram of Figure 10,

$$
\sigma_{1}=-1, \quad \sigma_{2}=+1, \quad \sigma_{3}=+1, \quad \sigma_{4}=-1, \quad \sigma_{5}=-1 .
$$

These data are used to show the types of vertex operators as

$$
\Gamma_{ \pm}^{\sigma}(\boldsymbol{x})= \begin{cases}\Gamma_{ \pm}(\boldsymbol{x}) & \text { if } \sigma=+1 \\ \Gamma_{ \pm}^{\prime}(\boldsymbol{x}) & \text { if } \sigma=-1\end{cases}
$$

Let us further introduce the auxiliary notations

$$
\beta^{(n)}=\left\{\begin{array}{ll}
\beta_{n} & \text { if } \sigma_{n}=+1, \\
{ }^{\mathrm{t}} \beta_{n} & \text { if } \sigma_{n}=-1,
\end{array} \quad Q_{m n}=Q_{m} Q_{m+1} \cdots Q_{n-1} .\right.
$$

With these notations, the fermionic expression of $Z_{\beta_{1} \cdots \beta_{N}}^{\alpha_{0} \alpha_{N}}$ read

$$
\begin{aligned}
Z_{\beta_{1} \cdots \beta_{N}}^{\alpha_{0} \alpha_{N}}= & q^{\left(1-\sigma_{1}\right) \kappa\left(\alpha_{0}\right) / 4} q^{\left(1+\sigma_{N}\right) \kappa\left(\alpha_{N}\right) / 4} s_{{ }^{\mathrm{t}} \beta_{1}}\left(q^{-\rho}\right) \cdots s_{{ }^{\mathrm{t}} \beta_{N}}\left(q^{-\rho}\right) \\
& \times\left\langle{ }^{\mathrm{t}} \alpha_{0}\right| \Gamma_{-}^{\sigma_{1}}\left(q^{-\beta^{(1)}-\rho}\right) \Gamma_{+}^{\sigma_{1}}\left(q^{{ }^{\mathrm{t}} \beta^{(1)}-\rho}\right)\left(\sigma_{1} Q_{1} \sigma_{2}\right)^{L_{0}} \cdots \\
& \times \Gamma_{-}^{\sigma_{N-1}}\left(q^{-\beta^{(N-1)}-\rho}\right) \Gamma_{+}^{\sigma_{N-1}}\left(q^{-{ }^{\mathrm{t}} \beta^{(N-1)}-\rho}\right)\left(\sigma_{N-1} Q_{N-1} \sigma_{N}\right)^{L_{0}} \\
& \times \Gamma_{-}^{\sigma_{N}}\left(q^{-\beta^{(N)}-\rho}\right) \Gamma_{+}^{\sigma_{N}}\left(q^{-{ }^{\mathrm{t}} \beta^{(N)}-\rho}\right)\left|\alpha_{N}\right\rangle
\end{aligned}
$$

In the case where $N=1$, this formula reduces to the fermionic expression (4.6) of the vertex weight itself. Starting from (4.6), one can prove this formula by induction. If $\alpha_{0}=\alpha_{N}=\emptyset$, one can use the commutation relations (5.10) to move $\Gamma_{-}^{\sigma}$ 's to the left and $\Gamma_{+}^{\sigma}$ 's to the right until they hit $\langle 0|$ and $|0\rangle$ and disappear. This yields the explicit formula

$$
\begin{aligned}
Z_{\beta_{1} \cdots \beta_{N}}^{\emptyset \emptyset} & =s{ }_{\beta_{1}}\left(q^{-\rho}\right) \cdots s{ }_{\beta_{N}}\left(q^{-\rho}\right) \\
& \times \prod_{1 \leq m<n \leq N} \prod_{i, j=1}^{\infty}\left(1-Q_{m n} q^{-{ }^{\mathrm{t}} \beta_{i}^{(m)}-\beta_{j}^{(n)}+i+j-1}\right)^{-\sigma_{m} \sigma_{n}}
\end{aligned}
$$

of Iqbal and Kashani-Poor [2]. 


\section{B Direct proof of two-leg cyclic symmetry}

As another application of the techniques used in Section 3, we present a direct proof of the identities (4.7) and (4.8) that amounts to the cyclic symmetry of two-leg vertices. Actually, these two identities are equivalent, and can be reduced to the following one:

$$
s_{\lambda}\left(q^{-\rho}\right) s_{\mu}\left(q^{-\lambda-\rho}\right)=\left\langle\mu\left|q^{-K / 2} \Gamma_{-}^{\prime}\left(q^{-\rho}\right) \Gamma_{+}^{\prime}\left(q^{-\rho}\right) q^{-K / 2}\right| \lambda\right\rangle .
$$

It is this identity that we prove here. Note that this identity implies the non-trivial relation

$$
s_{\lambda}\left(q^{-\rho}\right) s_{\mu}\left(q^{-\lambda-\rho}\right)=s_{\mu}\left(q^{-\rho}\right) s_{\lambda}\left(q^{-\mu-\rho}\right),
$$

from which the equivalence of (4.7) and (4.8) follows.

We prove (B.1) by generating functions. Namely, we construct generating functions of both sides by the Schur functions $s_{\mu}(\boldsymbol{x}), \boldsymbol{x}=\left(x_{1}, x_{2}, \ldots\right)$, and confirm that these generating functions are identical.

It is easy to calculate the generating function of the left side of (B.1). By the Cauchy identity

$$
\sum_{\mu \in \mathcal{P}} s_{\mu}(\boldsymbol{x}) s_{\mu}(\boldsymbol{y})=\prod_{i, j=1}^{\infty}\left(1-x_{i} y_{j}\right)^{-1}, \quad \boldsymbol{y}=\left(y_{1}, y_{2}, \ldots\right),
$$

of the Schur functions [28], the generating function of the left side of (B.1) can be expressed as

$$
\sum_{\mu \in \mathcal{P}} s_{\mu}(\boldsymbol{x}) s_{\lambda}\left(q^{-\rho}\right) s_{\mu}\left(q^{-\lambda-\rho}\right)=s_{\lambda}\left(q^{-\rho}\right) \prod_{i, j=1}^{\infty}\left(1-x_{i} q^{-\lambda_{j}+j-1 / 2}\right)^{-1} .
$$

On the other hand, constructing the generating function of the left side of (B.1) amounts to inserting $\Gamma_{+}(\boldsymbol{x})$ to the right of $\langle 0|$ as

$$
\begin{aligned}
& \sum_{\mu \in \mathcal{P}} s_{\mu}(\boldsymbol{x})\left\langle\mu\left|q^{-K / 2} \Gamma_{-}^{\prime}\left(q^{-\rho}\right) \Gamma_{+}^{\prime}\left(q^{-\rho}\right) q^{-K / 2}\right| \lambda\right\rangle \\
& =\left\langle 0\left|\Gamma_{+}(\boldsymbol{x}) q^{-K / 2} \Gamma_{-}^{\prime}\left(q^{-\rho}\right) \Gamma_{+}^{\prime}\left(q^{-\rho}\right) q^{-K / 2}\right| \lambda\right\rangle \\
& =\left\langle 0\left|\exp \left(\sum_{i, k=1}^{\infty} \frac{x_{i}^{k}}{k} J_{k}\right) q^{-K / 2} \Gamma_{-}^{\prime}\left(q^{-\rho}\right) \Gamma_{+}^{\prime}\left(q^{-\rho}\right) q^{-K / 2}\right| \lambda\right\rangle .
\end{aligned}
$$


The subsequent calculation is very similar to Section 3. One can use (3.6) and (3.8) to rewrite the last quantity as

$$
\begin{aligned}
& \left\langle 0\left|\exp \left(\sum_{i, k=1}^{\infty} \frac{x_{i}^{k}}{k} J_{k}\right) q^{-K / 2} \Gamma_{-}^{\prime}\left(q^{-\rho}\right) \Gamma_{+}^{\prime}\left(q^{-\rho}\right) q^{-K / 2}\right| \lambda\right\rangle \\
& =\left\langle 0\left|q^{-K / 2} \Gamma_{-}^{\prime}\left(q^{-\rho}\right) \Gamma_{+}^{\prime}\left(q^{-\rho}\right) \exp \left(\sum_{i, k=1}^{\infty} \frac{x_{i}^{k} q^{k / 2}}{k}\left(V_{0}^{(-k)}+\frac{1}{1-q^{k}}\right)\right) q^{-K / 2}\right| \lambda\right\rangle \\
& =\left\langle 0\left|\Gamma_{+}^{\prime}\left(q^{-\rho}\right) q^{-K / 2} \exp \left(\sum_{i, k=1}^{\infty} \frac{x_{i}^{k} q^{k / 2}}{k}\left(V_{0}^{(-k)}+\frac{1}{1-q^{k}}\right)\right)\right| \lambda\right\rangle .
\end{aligned}
$$

Note that the order of $\exp (\cdots)$ and $q^{K / 2}$ has been exchanged because $V_{0}^{(-k)}$ commutes with $q^{K / 2}$. By (3.1), the action of $\exp (\cdots)$ on $|\lambda\rangle$ can be expressed as

$$
\begin{aligned}
& \exp \left(\sum_{i, k=1}^{\infty} \frac{x_{i}^{k} q^{k / 2}}{k}\left(V_{0}^{(-k)}+\frac{1}{1-q^{k}}\right)\right)|\lambda\rangle \\
& =\exp \left(\sum_{i, k=1}^{\infty} \frac{x_{i}^{k} q^{k / 2}}{k} \sum_{j=1}^{\infty} q^{-k\left(\lambda_{j}-j+1\right)}\right)|\lambda\rangle \\
& =\prod_{i, j=1}^{\infty} \exp \left(\sum_{k=1}^{\infty} \frac{\left(x_{i} q^{-\lambda_{j}+j-1 / 2}\right)^{k}}{k}\right)|\lambda\rangle \\
& =\prod_{i, j=1}^{\infty}\left(1-x_{i} q^{-\lambda_{j}+j-1 / 2}\right)^{-1}|\lambda\rangle .
\end{aligned}
$$

Thus the generating function of the right side of (B.1) turns out to take such a form as

$$
\begin{aligned}
& \sum_{\mu \in \mathcal{P}} s_{\mu}(\boldsymbol{x})\left\langle\mu\left|q^{-K / 2} \Gamma_{-}^{\prime}\left(q^{-\rho}\right) \Gamma_{+}^{\prime}\left(q^{-\rho}\right) q^{-K / 2}\right| \lambda\right\rangle \\
& =\left\langle 0\left|\Gamma_{+}^{\prime}\left(q^{-\rho}\right) q^{-K / 2}\right| \lambda\right\rangle \prod_{i, j=1}^{\infty}\left(1-x_{i} q^{-\lambda_{j}+j-1 / 2}\right)^{-1} \\
& =q^{-\kappa(\lambda) / 2} s_{{ }}\left(q^{-\rho}\right) \prod_{i, j=1}^{\infty}\left(1-x_{i} q^{-\lambda_{j}+j-1 / 2}\right)^{-1}
\end{aligned}
$$

By (4.4), this coincides with (B.4). 


\section{References}

[1] M. Aganagic, A. Klemm, M. Mariño and C. Vafa, The topological vertex, Commun. Math. Phys. 254 (2005), 425-478. arXiv:hep-th/0305132.

[2] A. Iqbal and A.-K. Kashani-Poor, The vertex on a strip, Adv. Theor. Math. Phys. 10 (2006), 317-343. arXiv:hep-th/0410174.

[3] J. Bryan and D. Karp, The closed topological vertex via the Cremona transform, J. Algebraic Geom. 14 (2005), 529-542. arXiv:math/0311208 [math.AG].

[4] D. Karp, C. M. Liu and M. Mariño, The local Gromov-Witten invariants of configurations of rational curves, Geometry and Topology 10 (2006), 115-168. arXiv:math/0506488 [math.AG].

[5] P. Sułkowski, Crystal model for the closed topological vertex geometry, JHEP 0612 (2006), 030. arXiv:hep-th/0606055.

[6] M. Aganagic, R. Dijkgraaf, A. Klemm, M. Mariño and C. Vafa, Topological strings and integrable hierarchies, Comm. Math. Phys. 261 (2006), 451-516. arXiv:hep-th/0312085.

[7] R. Dijkgraaf and C. Vafa, Two dimensional Kodaira-Spencer theory and three dimensional Chern-Simons gravity, arXiv:0711.1932 [hep-th].

[8] S. Gukov and P. Sułkowski, A-polynomial, B-model, and quantization, JHEP 1202 (2012), 070. arXiv:1108.0002 [hep-th].

[9] T. Nakatsu and K. Takasaki, Melting crystal, quantum torus and Toda hierarchy, Commun. Math. Phys. 285 (2009), 445-468. arXiv:0710.5339 [hep-th].

[10] T. Nakatsu and K. Takasaki, Integrable structure of melting crystal model with external potentials, M.-H. Saito, S. Hosono and K. Yoshioka (eds.), New Developments in Algebraic Geometry, Integrable Systems and Mirror Symmetry, Adv. Stud. Pure Math. vol. 59, Mathematical Society of Japan, Tokyo, 2010, pp. 201-223. arXiv:0807.4970 [math-ph].

[11] K. Takasaki, Modified melting crystal model and Ablowitz-Ladik hierarchy, J. Phys. A: Math. Theor. 46 (2013), 245202. arXiv:1302.6129 [math-ph]. 
[12] K. Takasaki, Orbifold melting crystal models and reductions of Toda hierarchy, J. Phys. A: Math. Theor. 48 (2015), 215201. arXiv:1410.5060 [math-ph].

[13] A. Okounkov and R. Pandharipande, Gromov-Witten theory, Hurwitz theory, and completed cycles, Annals of Math. 163 (2006), 517-560. arXiv:math.AG/0204305.

[14] J. Harnad and A. Y. Orlov, Convolution symmetries of integrable hierarchies, matrix models and $\tau$ functions, P. Deift and P. Forrester (eds.), Random Matrix Theory, Interacting Particle Systems, and Integrable Systems, MSRI Publications, vol. 65, Cambridge University Press, 2014, pp. 247-275. arXiv:0901.0323 [math-ph].

[15] A. K. Kashani-Poor, The wave function behavior of the open topological string partition function on the conifold, JHEP 04 (2007), 004. arXiv:hep-th/0606112

[16] S. Hyun and S.-H. Yi, Non-compact topological branes on conifold, JHEP 0611 (2006), 075. arXiv:hep-th/0609037.

[17] J. Zhou, Quantum mirror curves for $\mathbb{C}^{3}$ and the resolved conifold, arXiv:1207.0598 [math.AG].

[18] K. Takasaki, Remarks on partition functions of topological string theory on generalized conifolds, arXiv:1301.4548 [math-ph].

[19] L. Faddeev and A. Yu. Volkov, Abelian current algebra and the Virasoro algebra on the lattice, Phys. Lett. B315 (1993), 311-318, arXiv:hep-th/9307048.

[20] L. D. Faddeev and R. M. Kashaev, Quantum dilogarithm, Mod. Phys. Lett. A9 (1994), 427-434, arXiv:hep-th/9310070.

[21] A. Iqbal, C. Kozçaz and C. Vafa, The refined topological vertex, JHEP 0910 (2009), 069. arXiv:hep-th/0701156.

[22] M. Taki, Refined topological vertex and instanton counting, JHEP 803 (2008), 048. arXiv:0710.1776 [hep-th].

[23] A. Okounkov, N. Reshetikhin and C. Vafa, Quantum Calabi-Yau and classical crystals, P. Etingof, V. Retakh and I.M. Singer (eds.), The unity of mathematics, Progr. Math. vol. 244, Birkhäuser, 2006, pp. 597-618. arXiv:hep-th/0309208. 
[24] I. P. Goulden and D. M. Jackson, Transitive factorisations into transpositions and holomorphic mappings on the sphere, Proc. Amer. Math. Soc. 125 (1997), 51-60.

[25] M. E. Kazarian and S. K. Lando, An algebro-geometric proof of Witten's conjecture, J. Amer. Math. Soc. 20 (2007), 1079-1089. arXiv:math/0601760.

[26] A. Okounkov and N. Reshetikhin, Correlation function of Schur process with application to local geometry of a random 3-dimensional young diagram, J. Amer. Math. Soc. 16, (2003), 581-603. arXiv:math/0107056.

[27] J. Bryan and B. Young, Generating functions for coloured 3D Young diagrams and the Donaldson-Thomas invariants of orbifolds, Duke Math. J. 152 (2010), 115-153. arXiv:0802.3948 [math.CO].

[28] I. G. Macdonald, Symmetric functions and Hall polynomials, Oxford University Press, 1995.

[29] T. Miwa, M. Jimbo and E. Date, Solitons: Differential equations, symmetries, and infinite-dimensional algebras, Cambridge University Press, 2000 .

[30] J. Zhou, A conjecture on Hodge integrals, arXiv:math.AG/0310282.

[31] Y. Konishi and S. Minabe, Flop invariance of the topological vertex, Int. J. Math. 19 (2008), 27-45. arXiv:math/0601352.

[32] T. Eguchi and H. Kanno, Geometric transitions, Chern-Simons gauge theory and Veneziano type amplitudes, Phys. Lett. B585 (2004), 163172. arXiv:hep-th/0312223.

[33] K. Nagao, Non-commutative Donaldson-Thomas theory and vertex operators, Geometry and Topology 15 (2011) 1509-1543. arXiv:0910.5477 [math.AG].

[34] P. Sułkowski, Wall-crossing, free fermions and crystal melting, Comm. Math. Phys. 301 (2011), 517-562. arXiv:0910.5485 [hep-th]. 